\title{
Note textuelle sur un (problème de) lieu géométrique dans les Météorologiques d'Aristote \\ (III. 5, 375 b 16 - 376 b 22)
}

Bernard Vitrac

\section{Introduction}

La présente note porte seulement sur une petite partie du Chapitre III. 5 des Météorologiques d'Aristote, qu'elle compare, après Tannery, Heath, et Knorr, à la preuve d'un théorème des Lieux plans d'Apollonius rapportée par Eutocius. Son objectif n'est donc pas l'étude de la célèbre théorie aristotélicienne de l'arc-en-ciel ${ }^{1}$ mais une analyse textuelle et mathématique rendue nécessaire parce que l'authenticité et la cohérence de ladite portion peuvent être contestées. Dans cet article, j'utilise un certain nombre de résultats que j'ai obtenu en étudiant l'histoire du texte des Éléments d'Euclide, étant entendu que rien ne prouve a priori la légitimité d'une telle utilisation. Je commence par résumer l'état historiographique de la question. J'examine ensuite la portée et la pertinence de deux critères proposés par P. Tannery, ce qui oblige à regarder d'un peu plus près la manière dont Aristote formule certains arguments de style mathématique. Cet examen permet de proposer une (en fait plusieurs) reconstruction(s) probable(s) du texte aristotélicien. Je la confronte avec la preuve conservée par Eutocius dans une troisième partie. L'annexe inclut le texte de ladite preuve et sa traduction française; j'y ai ajouté un extrait de la Collection mathématique de Pappus que j'utilise à plusieurs reprises ainsi qu'une tentative de restitution de la preuve d'Apollonius².

\section{Historiographie}

1. Dans l'article qu'il a consacré au Chapitre 5 du Livre III des Météorologiques d'Aristote, publié en 1886 dans la Revue de philologie, de littérature et d'histoire anciennes ${ }^{3}$, Paul Tannery faisait le rapprochement, apparemment pour la première fois, entre une portion de l'analyse aristotélicienne de la forme de l'arc-en-ciel et un théorème de la théorie des Lieux Plans, exposé par Eutocius lequel fait référence à un traité d'Apollonius appartenant à la collection dite du Lieu analysé (é $\nu$ Tô

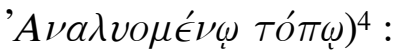

« Deux points étant donnés et un rapport donné entre deux droites inégales, il est possible de décrire un cercle dans le plan de sorte que les droites fléchies à partir des points donnés jusqu'à la circonférence du cercle aient le même rapport que le rapport donné $\gg^{5}$.

Ledit cercle est traditionnellement appelé « cercle d'Apollonius » par les historiens des mathématiques, et je continuerai à faire de même. En outre je désignerai le résultat lui-même comme « théorème d'Apollonius » quand il n'y a pas d'ambiguïté.

La même collection d'ouvrages géométriques est également mentionnée par Pappus, dans le

\footnotetext{
1 Pour une telle étude voir l'article d'Anne Merker, "Aristote et l'arc-en-ciel : enjeux philosophiques et étude scientifique", pp. 00-00, qui donne aussi de nombreuses références bibliographiques. J'ai entrepris ce travail à l'occasion des séances du Séminaire de l'équipe Savoirs et Textes, CNRS-Université de Lille III, animé par S. Rommevaux, séances consacrées à la théorie aristotélicienne de l'arc-en-ciel. Je remercie les participants pour leurs stimulantes remarques et tout particulièrement $\mathbf{M}^{\mathrm{me}}$ Merker : sans sa sollicitation, je ne l'aurai pas entrepris. Nos deux articles sont donc en quelque sorte complémentaires quoique nous ayons veillé à maintenir l'autonomie de nos contributions respectives. Comme le lecteur s'en rendra aisément compte en consultant les notes infrapaginales il tirera certainement un avantage à lire l'un et l'autre.

2 Pour le chapitre III. 5 des Météorologiques, outre qu'il s'agit d'un texte très facilement accessible, le lecteur en trouvera le texte grec tel que le donnent les manuscrits, la traduction française et des notes explicatives par $\mathbf{M}^{\mathrm{me}} \mathbf{M e r k e r}$ pp. 00-00. Pour assurer l'autonomie de lecture les passages discutés en détail ici sont reproduits dans le corps de l'article.

3 [Tannery, MSc, 1929]. Références complètes dans la bibliographie à la fin de cet article.

4 V. [Apollonii Pergaei ..., 1974], t. II, p. 180, 1. 11-p. 184, 1. 20. V. infra, Annexe II. Dans ce qui suit, j'utilise l'abréviation [Eut. in Apoll.] pour l'édition par Heiberg du commentaire d'Eutocius.

5 [Eut. in Apoll.], p. 180, 1. 13-17 et Annexe II. En fait l'énoncé original d'Apollonius était peut-être double et incluait non seulement l'existence du lieu cherché mais aussi son unicité; v. la restitution que je propose dans l'annexe III.
} 
Livre VII de sa Collection mathématique, et si l'on en croit le sommaire que ce dernier propose pour le traité des Lieux-Plans ${ }^{6}$, le " théorème d'Apollonius » constituait une partie du deuxième résultat du Livre II dudit traité :

«Si des droites menées de deux points donnés se brisent, et si les carrés décrits sur ces droites diffèrent d'une aire donnée, le point est lié à une droite donnée de position. D'autre part si ces droites sont en rapport donné, le point sera lié soit à une droite ${ }^{7}$, soit à une circonférence $»^{8}$.

2. Tannery commente le texte du Stagirite dans le but de détecter des passages suspects à l'aide de différents critères stylistiques sur lesquels je reviendrai (Partie II, A, infra). Sa conclusion est qu'une portion du développement strictement géométrique destiné à établir la première assertion

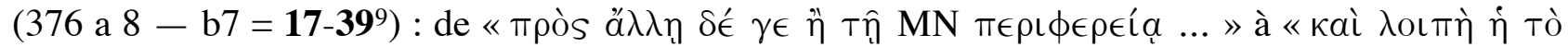
MH про̀s MK ») est inauthentique. Tannery affirme qu'un interpolateur s'est (fortement) inspiré d'une démonstration géométrique existante pour insérer dans le texte d'Aristote une preuve qualifiée d'"accessoire", correspondant à la première partie de la démonstration d'Apollonius rapportée par Eutocius ${ }^{10}$. Son analyse suggère l'idée d'un "montage" assez simple à partir de deux textes, celui des Météorologiques - son objectif est de le dégager - et un traité géométrique. Il en résulte donc un découpage du texte en trois parties $(\mathrm{A}-\mathrm{B}-\mathrm{C})$ dont seules les extrêmes $(\mathrm{A}, \mathrm{C})$ sont globalement authentiques, même si l'interpolateur y a également introduit quelques modifications et "améliorations" ponctuelles.

3. Tannery ne croit pas que la source de l'interpolateur soit le texte d'Apollonius lui-même : il est persuadé que l'interpolation est antérieure à l'auteur des Coniques. Il considère qu'Aristote a fait allusion à une proposition de géométrie plane bien connue à l'époque - quoiqu'elle ne figure pas dans les Éléments d'Euclide - qui « faisait partie de la théorie des lieux plans, déjà élaborée par Hermotime de Colophon, entre autres ${ }^{11}$ et que cette allusion a provoqué l'interpolation centrale. Il n'y a évidemment rien d'invraisemblable à ce qu'un tel théorème ait été énoncé avant Euclide et même avant Aristote.

Mais sa possible attribution à Hermotime, « entre autres », est passablement incertaine. Cet auteur est mentionné dans un unique témoignage antique, à savoir le célèbre résumé de l'histoire de la géométrie rapporté par Proclus dans son Commentaire au premier Livre des Éléments d'Euclide ${ }^{12}$

«Et Hermotime de Colophon fit progresser sur de nombreux points les résultats procurés

\footnotetext{
${ }^{6}$ V. Pappi Alexandrini ..., vol. 2, p. 660, 1. 17- p. 670, 1. 2 (dans ce qui suit, j’utilise l'abréviation [Papp., Coll. math.]). Cf. [Ver Eecke, 1982], pp. 495-501.

${ }^{7}$ C'est bien entendu le cas si le rapport donné est le rapport identique; les deux droites égales se brisent en un point situé sur la médiatrice du segment qui joint les deux points donnés. Ce cas de figure est exclu dans la citation d'Eutocius puisqu'il suppose deux droites données inégales.

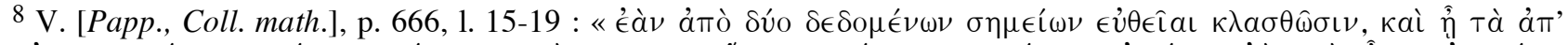

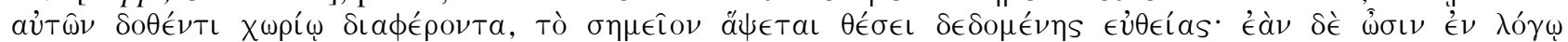

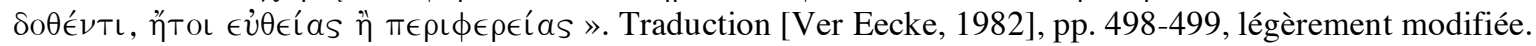

${ }^{9}$ Pour la commodité des renvois le texte du chapitre III. 5 est divisé en assertions numérotées dans Merker, pp. 00-00. J'utiliserai le même système.

10 [Tannery, MSc, 1929], p. 57 : «En fait, l'interpolateur montre comment, si le rapport MH : MK est donné avec les points $\mathrm{H}$ et $\mathrm{K}$, on peut construire une circonférence dont les points soient tels que le rapport de leurs distances à $\mathrm{H}$ et $\mathrm{K}$ soit égal au rapport donné; sa démonstration présente la plus grande analogie avec la partie correspondante du théorème d'Apollonius sur le même objet, tel qu'Eutocius l'a extrait des Lieux plans du géomètre de Perge et inséré dans son commentaire sur les Coniques (...) Au contraire, l'autre partie du même théorème - à savoir que, si un point M est tel que ses distances à $\mathrm{H}$ et $\mathrm{K}$ soient dans le rapport donné, ce point sera sur la circonférence construite, — n'est pas démontrée par l'interpolateur ».

11 [Tannery, MSc, 1929], p. 57.

${ }^{12}$ V. Procli Diadochi in primum Euclidis ..., pp. 64. 16-68. 6 (dans ce qui suit, j'utilise l'abréviation [Pr., in. Eucl. I]). La plupart des historiens rattachent ce texte, au moins en partie, aux Histoires géométriques d'Eudème de Rhodes, disciple d'Aristote, malheureusement perdues. Le Résumé est le fragment N¹33 dans [Wehrli, 1969], pp. 54-56).
} 
auparavant par Eudoxe et Théétète, découvrit beaucoup de résultats des éléments et en composa certains des lieux $»^{13}$.

4. De ce texte on déduit qu'Hermotime était un continuateur d'Eudoxe - et donc un contemporain d'Aristote - , qu'il composa ou regroupa certains résultats de la théorie des lieux. Malheureusement le texte n'indique pas que ceux-ci incluaient précisément le théorème d'Apollonius, ni même pace Tannery - qu'il s'agissait de lieux plans. Or cette époque est celle où s'est également

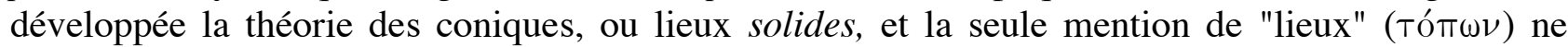
suffit pas à trancher. On connaît les titres d'ouvrages ${ }^{14}$, l'un d'Euclide : "Les Lieux à la surface »

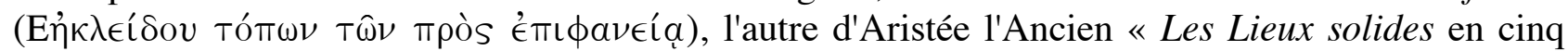

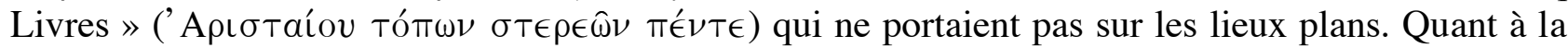
mention «entre autres », rien ne la justifie. En particulier le Résumé de Proclus - si l'on veut bien le considérer comme une source fiable pour l'histoire de la géométrie préeuclidienne ${ }^{15}$ - ne rattache la théorie des lieux à aucun des dix-neuf autres mathématiciens qui y sont cités. En fait, l'argument principal en faveur de l'attribution proposée par Tannery réside précisément dans le fait qu'Aristote cite apparemment ce résultat. Or, l'une des questions que soulève l'étude philologique de Tannery est de savoir si le Stagirite se réfère ou non au théorème dit d'Apollonius.

5. Le travail de Tannery a eu apparemment peu d'écho ${ }^{16}$. Thomas Little Heath lui-même croit avoir été le premier - dans sa traduction commentée des Éléments d'Euclide ${ }^{17}$ - à rapprocher nos deux textes ${ }^{18}$. Dans son Mathematics in Aristotle, il maintient que le passage des Météorologiques contient et prouve tout à fait correctement (« quite correctly ») un théorème donné à peu près exactement de la même manière (« in almost exactly the same way ») par Apollonius. Comme chez Tannery, cette attestation aristotélicienne prouve que le résultat avait été découvert - et prouvé de la même manière - avant l'époque d'Apollonius. Il y a donc accord partiel avec Tannery — sans le savoir - du point de vue de l'histoire générale des mathématiques, et aussi sur le fait qu'Aristote ne produisait que la première partie de la preuve ${ }^{19}$.

Les différences essentielles avec Tannery tiennent à ce qu'il n'y a ni remise en cause de l'intégrité textuelle du chapitre - la seule correction proposée concerne une faute de lettrage dans une proportion, correction qui n'a évidemment rien à voir avec le rejet global de la portion centrale que propose Tannery -, ni remise en cause de son authenticité aristotélicienne. Ce n'est pourtant pas l'habitude du Stagirite d'inclure d'aussi longs développements purement géométriques et, à cet égard, le chapitre III. 5 des Météorologiques est unique en son genre dans l'ensemble des traités globalement authentiques d'Aristote.

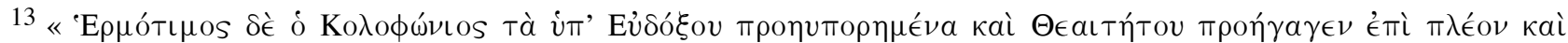

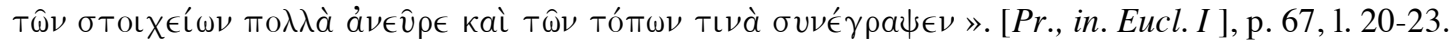

14 V. [Papp., Coll. math.], p. 636, 1. 23-24 et [Ver Eecke, 1982], p. 479.

15 Sur ce sujet délicat, v. [Vitrac, 1996a], pp. 35-38, 40-45 et [Vitrac, 2001], pp. 98-102.

16 Même chez les auteurs de langue française. Dans sa traduction des Météorologiques (v. [Aristote, Tricot, 1976]), J. Tricot ne mentionne pas ce travail. Il n'émet aucune réserve d'authenticité à propos du texte de III. 5; de même G. G. Granger consacre quelques pages à la théorie aristotélicienne de l'arc-en-ciel ([Granger, 1976], pp. 319-332) sans s'interroger sur l'intégrité de ce chapitre. Quant au dernier éditeur français du traité, P. Louis, il cite l'article de Tannery (v. [Aristote, Louis, 1982], vol. 2, p. 20, n. 2), il souligne les incertitudes textuelles de la démonstration aristotélicienne (ibid., n. 3, p . 153), mais il se garde bien de prendre parti. Dans sa thèse, G. Picolet mentionne également l'article de Tannery, mais sur des points mineurs ([Picolet, 1982], n. 105, p. 285; n. 138, p. 291; n. 195, p. 300), sans se prononcer sur la reconstruction que celui-ci a proposée qui n'est d'ailleurs pas compatible avec le jugement d'authenticité globale auquel aboutit Picolet, proche de celui qu'avait soutenu Heath avant lui.

17 « But the construction was not discovered by Apollonius, it belongs to a much earlier date since it appears in exactly the same form in Aristotle Meteorologica, III. 5, 376 a $3 s q$... ; [Euclid, 1956], vol. II, pp. 198-199. Il s'agit de la réédition de la $2^{\mathrm{e}}$ édition, Cambridge University Press, London, 1926. La première édition était de 1908.

18 [Heath, 1949], p. 182 : « I called attention to this remarkable coïncidence in my edition ... But I have not seen it noticed either before or since; and I think that the more recent writers on our passage have lost much by the omission ».

${ }^{19}$ Fait évoqué de manière quelque peu sibylline un peu plus loin ([Heath, 1949], p. 188) : «Apollonius completes his proposition ...».
} 
6. La position de Heath est donc plutôt difficile à soutenir sans être nuancée. C'est ce qu'a fait récemment W. R. Knorr ${ }^{20}$. Il décrit la relation entre les deux textes d'une manière un peu plus précise :

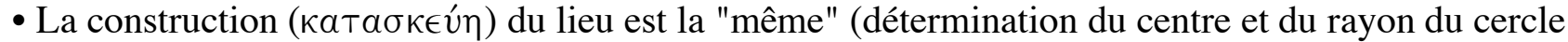
cherché), par contraste avec une construction alternative (détermination dudit cercle par son diamètre), assez naturelle pour qui connaît la Proposition VI. 3 des Éléments d'Euclide et son extension à la bissectrice extérieure d'un angle ${ }^{21}$.

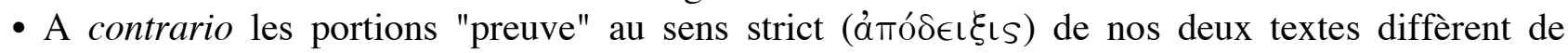
manière significative.

Comme Heath, il ne remet absolument pas en cause l'intégrité textuelle du texte des Météorologiques. La démonstration lui paraît embrouillée ("muddled"22) mais il ne la dit pas "corrompue". Bien qu'il déplore le fait que l'analyse de Heath ait été longtemps ignorée 23 , lui-même ne connaît pas l'article de Tannery et, de ce fait, n'envisage pas, au moins à titre d'hypothèse, de contester l'authenticité de certaines portions du texte.

7. Dans la mesure où il lui paraît peu probable qu'Aristote soit l'auteur d'un tel chapitre, dans la mesure aussi où la démonstration géométrique fait appel à des principes ou hypothèses qui lui paraissent incompatibles avec la doctrine aristotélicienne habituelle - principe du rayon visuel émis par l'œil; existence de la sphère "météorologique"24 —, Knorr est amené à introduire un hypothétique "auteur aristotélicien" 25 pour ces preuves, sans bien préciser d'ailleurs ce qu'on doit lui attribuer : le chapitre 5 du Livre III, ou bien les seules analyses géométriques (Ch. 3 et 5 incluant la détermination de la forme du halo), ou bien encore l'ensemble des descriptions concernant les phénomènes de réflexion. Toujours est-il qu'il postule une composition par strates pour le traité des Météorologiques, au moins pour le Livre III. Selon Knorr, l'auteur de nos démonstrations aurait été motivé par la nouveauté et le caractère frappant du résultat que constituait le théorème de lieu ${ }^{26}$. Dans cette perspective, la description de l'arc-en-ciel devient un simple prétexte pour avoir l'opportunité de présenter cette démonstration géométrique ${ }^{27}$.

8. Pour résumer ce très sommaire survol historiographique, malgré Heath et la prudente abstention de la plupart des éditeurs des Météorologiques, il parait bien difficile de recevoir le texte de notre chapitre III. 5 comme authentiquement aristotélicien. Cela dit, la solution de Knorr ne me paraît pas totalement satisfaisante, ne serait-ce que dans sa façon d'aborder la question, en isolant le chapitre III. 5 de son contexte. Ainsi les hypothèses du rayon visuel émis par l'œil et de la sphère météorologique sont également mises en œuvre dans la démonstration de la forme circulaire du halo (Ch. III. $3^{28}$ ) et la première de ces hypothèses intervient même pour rendre compte de propriétés non géométriques de l'arc-en-ciel (Ch. III. 4) ${ }^{29}$ ainsi que dans l'explication des parhélies et des raies solaires $\left(\mathrm{Ch}\right.$. III. $\left.6^{30}\right)$. En somme, s'il fallait suivre l'hypothèse de Knorr, c'est la quasi-totalité du

\footnotetext{
${ }^{20}$ V. [Knorr, 1986], pp. 102-108, en particulier p. 103.

21 Cette construction a été proposée par Simson dans son édition des Éléments (1756). V. [Euclid, 1956], vol. II, pp. 197-198; [Knorr, 1986], pp. 104-105; [Euclide, 1994], pp. 164-167.

22 [Knorr, 1986], n. 10, p. 140.

23 [Knorr, 1986], n. 9, p. 139.

24 Autrement dit une sphère dont la surface porte le soleil (ou la lune) et les nuages, ce qui est incompatible avec la distinction aristotélicienne cardinale entre mondes supralunaire et sublunaire.

25 [Knorr, 1986], pp. 107-108.

26 [Knorr, 1986], pp. 106-107.

27 [Knorr, 1986], p. 108.

28 V. Meteor., III. 3, 372 b16-18; 372 b34-373 a5; 373 a16-19 et supra, Merker, pp. 00.

${ }^{29}$ V. Meteor., III. 4, 373 a32-b10; 373 b13-17; 374 b21-24; 375 b12-15 et supra, Merker, pp. 00.

30 V. Meteor., III. 6, 377 a29-b13; 377 b17-22; 377 b27-378 a11.
} 
Livre III des Météorologiques qu'il faudrait déclarer apocryphe ${ }^{31}$. Notre regretté collègue paraît grandement sous-estimer l'importance des problèmes de "réflexion" dans l'ensemble des Météorologiques. Plusieurs témoignages aristotéliciens soulignent que les explications en termes de réflexion du rayon visuel (ce que les Anciens nommeront ensuite «catoptrique ») étaient somme toute assez récentes; ils suggèrent que ce nouveau champ méritait toute l'attention du Stagirite, quelles que soient les réserves qu'il pouvait éprouver pour les principes de base de ce genre d'études ${ }^{32}$.

9. Finalement l'hypothèse de Tannery - en termes d'interpolations - me paraît plus économique, même s'il est nécessaire de la compléter sur plusieurs points. D'ailleurs, quand bien même on l'accepterait - au moins au titre d'explication partielle - , il resterait à déterminer l'extension de la (ou des) partie(s) non authentique(s) et cela est très important pour la question qui nous intéresse. Supposons, par exemple, que l'on rejette la portion 376 a1-376 b12 (12-41); nous obtenons un texte très allégé, une démonstration très simple pour l'affirmation (4), du même type que celle proposée plus haut dans le traité pour le halo (III. 3, 373 a 1-21). Mais, si tel est le cas, plus rien ne garantit qu'Aristote connaissait le théorème de lieu, puisque nous ne trouvons plus ni démonstration de, ni même allusion à, ce théorème. Même si elles n'ont rien d'invraisemblable, les conclusions historiques générales de Tannery et Heath, dans une telle hypothèse, n'auraient plus de support textuel.

\footnotetext{
${ }^{31}$ Lui-même est réticent devant une telle hypothèse extrême ([Knorr, 1986], p. 108). G. Picolet a repris en détail les arguments pro et contra l'hypothèse d'inauthenticité globale du L. III (v. [Picolet, 1982], pp. 69-74, 75-92). Son étude montre bien que cette hypothèse est très peu vraisemblable et ne résout à peu près aucun des problèmes que soulèvent les particularités de cette section du traité. Il envisage ensuite (op. cit., pp. 92-111; cf. aussi p. 189) l'hypothèse que les démonstrations géométriques concernant le halo et l'arc-en-ciel seules soient inauthentiques, dans la mesure où elles sont apparemment absentes de la traduction arabe traditionnellement attribuée à Yahyâ ibn al-Bitrîq et dans la traduction arabo-latine de Gérard de Crémone (op. cit., pp. 95-97). Elles existaient toutefois déjà dans la tradition directe grecque à l'époque d'Alexandre d'Aphrodise ( $\mathrm{II}^{\mathrm{e}} \mathrm{s}$. de notre ère). Picolet écarte également cette hypothèse, mais les raisonnements qu'il développe parviennent seulement à réfuter la thèse d'un faussaire ayant délibérément altéré le texte du Stagirite. Comme nous le verrons, d'autres hypothèses, moins "brutales", sont possibles.

32 V. Meteor., I. 3, 340 a24-32 (réflexion de la lumière des astres par la terre); I. 5, 342 b1-14 (apparitions colorées); I. 6, 342 b35-343 a20; 343 a26-30; 343 b4-7; I. 7, 344 b1-18 (explication de la queue des comètes par Hippocrate de Chio et son disciple Eschyle; critique de cette explication; I. 8, 345 b9-31 (explication de la Voie lactée, sans doute par les mêmes auteurs et critique); I. 12, 348 a14-20 (comme I. 3, pour expliquer la formation de la grêle); II. 9 370 a10-21 (explication de l'éclair selon Clidème); III. 2, 372 a16-21; 372 a29-b9 ainsi que les références des notes 28-30 supra; cf. De Sensu, Ch. 2, 438 a5-12 (v. aussi Merker, pp. 00); De anima, III, 12, 435 a5-10.
} 


\section{Les critères philologiques de Tannery et les reconstructions possibles du texte aristotélicien}

\section{A. Les critères de Tannery}

1. Outre les considérations de bon sens sur l'objectif d'Aristote dans ce passage, le souci d'une cohérence linguistique minimale, Tannery utilise essentiellement deux critères formels :

a. L'utilisation de formules "complexes" de désignation des objets géométriques « le [point] auprès duquel (ou sur lequel) [est] $\mathrm{A} »(T o ̀) \epsilon ' \hat{\dot{\omega}} \mathrm{A})$, « la [droite] auprès de laquelle (ou sur laquelle) [est] $\mathrm{B} \Gamma \gg\left(\dot{\eta} \epsilon \phi^{\prime} \hat{\eta} \mathrm{B} \Gamma\right)$, que l'on rencontre, selon Tannery, seulement dans Aristote et dans le fragment de son disciple Eudème sur la quadrature des lunules par Hippocrate de $\mathrm{Chio}^{33}$, au lieu des formules simples «le [point] $\mathrm{A}$ » (Tò $\mathrm{A})$, « la [droite] $\mathrm{B} \Gamma$ » $(\dot{\eta} \mathrm{B} \Gamma)$ que l'on trouve dans les textes des géomètres de l'époque hellénistique, Euclide, Archimède, Apollonius, du moins tels qu'ils nous ont été transmis (Cf. infra, B, §§ 7-9). Quand Tannery rédige cet article le critère en question avait déjà été utilisé par Allmann, Usener et lui-même ${ }^{34}$ pour isoler le texte d'Eudème-Hippocrate des gloses de Simplicius qui a transmis ce fragment. Tannery souligne lui-même les limites de ce critère : les copistes ont pu simplifier ou, à l'inverse restituer, à certains endroits une notation qu'ils identifiaient certainement comme "archaïsante" 35 . Reprenons, à titre d'exemple, le passage mentionné supra. Le texte grec est :

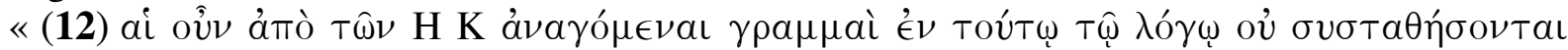

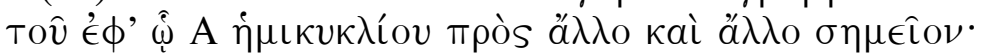

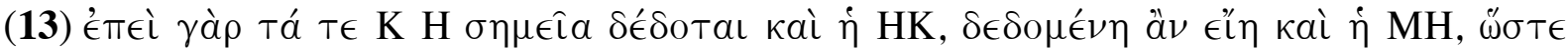

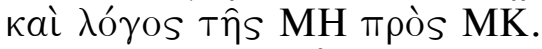

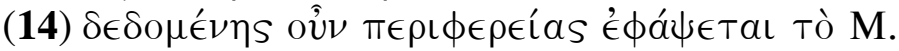

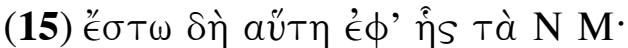

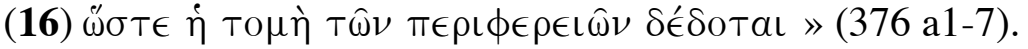

Le critère en question commande à Tannery de conserver ce passage à cause des deux occurrences de la formule complexe (soulignées en caractères gras ${ }^{36}$ ). Il rejette la précision quelque peu superfétatoire «ámò $\tau \hat{\omega} v \mathrm{H} \mathrm{K}$ ». Il rejette également la séquence explicative formulée dans la terminologie des Données d'Euclide $(\mathbf{1 3})^{37}$ et à l'aide des seules notations simples. Sans doute considère-t-il « Tò $\mathrm{M} »(\mathbf{1 4})$ comme le résultat d'une simplification.

b. Quant au second critère, il s'agit de l'utilisation de la formule non canonique : « ce que A est relativement à $\mathrm{B}, \Gamma$ l'est relativement à $\Delta »\left(\check{o} \pi \epsilon \rho \dot{\eta} A \pi \rho \grave{o} \varsigma \tau \dot{\eta} \nu B, \dot{\eta} \Gamma \pi \rho \grave{s} \varsigma \tau \grave{\eta} \nu \Delta^{38}\right)$ pour énoncer

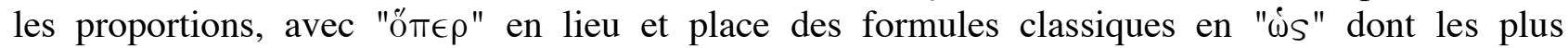
répandues sont « comme $\mathrm{A}$ est relativement à $\mathrm{B}$, ainsi est $\Gamma$ relativement à $\Delta$ » (ís ì $\mathrm{A} \pi \rho$ òs Tìv

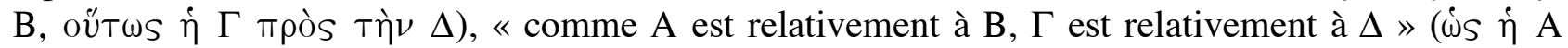

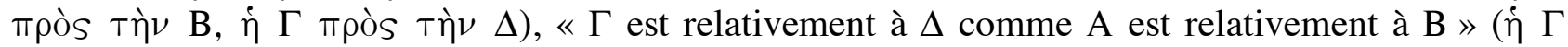

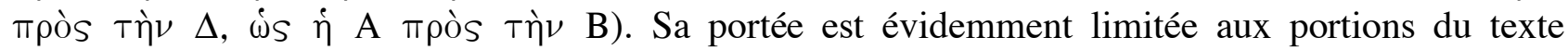
incluant des proportions. Ainsi, malheureusement, dans le second cas de figure (astre au-dessus de l'horizon), Aristote se contente de décrire un schéma, et ne fait pas appel à la théorie des proportions, excepté dans la formulation de contrevariance : «car plus le point $\mathrm{H}$ est haut, plus le

\footnotetext{
${ }^{33}$ Fragment $\mathrm{N}^{\circ} 140$ ([Wehrli, 1969], pp. 59-66) des Histoires géométriques d'Eudème de Rhodes.

34 [Tannery, $M S c, 1912]$.

35 [Tannery, $M S c$, 1929], pp. 52-53.

36 Dans le texte grec du ch. III. 5 (v. Merker, pp. 00-00), les occurrences de cette formule sont, comme ici, mises en gras pour en faciliter le repérage et en percevoir la répartition.

37 Tricot, à l'inverse, y voit la démonstration de l'affirmation précédente; pour lui (14) est la conclusion de cette séquence.

38 Dans le texte grec du ch. III. 5 (v. Merker, pp. 00-00), les occurrences de cette formule sont, comme ici, mises en italique pour en faciliter le repérage et en percevoir la répartition.
} 
pôle et le centre du cercle seront bas $»^{39}$. Quoi qu'il en soit, si l'on prend ce critère au sérieux, l'idiosyncrasie de la formule en " о̆ $\pi \epsilon \rho$ " exclut que l'interpolation - si c'en est une - soit le travail d'un géomètre hellénistique, ou, a fortiori, de l'Antiquité tardive ${ }^{40}$.

2. On pourrait même être tenté d'utiliser ce second critère à l'inverse de ce qu'en fait Tannery. Pourquoi Aristote - si le chapitre est de lui - devrait-il écrire les proportions comme Euclide ? Pourquoi faudrait-il postuler que l'écriture mathématique à l'époque d'Aristote était déjà complètement uniformisée et identique, sur ce point, à celle des géomètres de l'époque hellénistique, alors qu'elle est censée ne pas l'être selon le critère a ? Tannery n'en dit rien, mais sa conviction tient sans doute au résultat obtenu quand on reprend le texte du chapitre III. 5 et qu'on le balaie selon ces deux critères : grosso modo, là où apparaissent les formules complexes de lettrages, on ne trouve pas la forme non canonique en " ó $\pi \epsilon \rho$ " pour énoncer les proportions; là où celle-ci apparaît, les formules de lettrage simples sont très largement majoritaires. La coordination de ces deux observations suffit à suggérer, au moins dans les grandes lignes, la division tripartite proposée par Tannery.

3. L'utilisation du critère (a) ne se fait pas non plus sans problèmes. L'hypothèse implicite de Tannery est que le passage central controversé est le résultat du travail d'un (unique) interpolateur. S'il ne se soucie guère de sa cohérence intellectuelle, il postule, de la part de cet intervenant, une cohérence notationnelle. Mais on peut imaginer que le texte tel qu'il nous est parvenu soit le résultat d'une combinaison entre le texte originel d'Aristote et de multiples annotations marginales qui l'accompagnaient, lesquelles peuvent avoir des origines et des âges divers. Or, quand une annotation marginale porte sur une phrase spécifique d'un texte mathématique ancien, elle en reproduit généralement les notations. Alexandre, par exemple, au $\mathrm{II}^{\mathrm{e}}$ siècle de notre ère, utilise encore parfois la notation "archaïsante" du texte qu'il commente ${ }^{41}$. Si tel est le cas dans notre extrait, cela affaiblit la valeur du critère de Tannery.

4. Autre difficulté : l'examen détaillé de l'argument géométrique (que ne fait pas Tannery) montre qu'il y a, non pas deux, mais trois notations : à six reprises on trouve en effet une formule en quelque sorte intermédiaire, plus simple que la formule complexe "archaïsante", mais procédant du même esprit : « $\dot{\eta}$ Tò $\mathrm{A} »$, sans doute pour désigner « la [droite sur laquelle est] la lettre $\mathrm{A} »^{42}$. La première occurrence $(\dot{\eta}$ Tò $B ; 376$ a16) pourrait laisser croire qu'il s'agit d'une précision rendue nécessaire par l'introduction des notations additives par concaténation : $\mathrm{BZ}, \Delta \mathrm{Z}$ pour $\mathrm{B}+\mathrm{Z}, \Delta+\mathrm{Z}$, afin d'éviter qu'un copiste, lisant $\dot{\eta}$ B, n'imagine qu'il faille alors restituer $\dot{\eta}$ BZ. Mais plusieurs des autres occurrences s'appliquent à des droites désignées d'emblée et sans ambiguïtés par deux lettres. L'explication suggérée n'est donc pas suffisante.

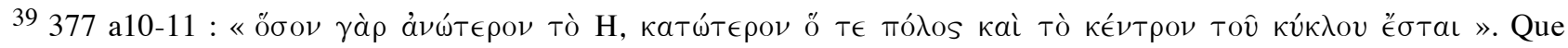
cette formule soit aussi à comprendre comme une indication de proportionnalité, c'est ce que montre bien le commentaire qu'en fait Alexandre (Commentaria in ..., pp. 171, 1. 21-172, 1. 6; dans ce qui suit j'utilise l'abréviation [Alex. in Meteor.]).

40 J'ajoute que je n'ai trouvé aucun mathématicien, hellénistique ou de l'Antiquité tardive, qui fasse usage de cette formule.

${ }^{41}$ V. [Alex. in Meteor.], p. 162, 1. 30-31; p. 164, 1. 18.

42 Pour la troisième de ces occurrences (376 a26) l'article "Tò" est omis dans certains mss, mais il y a unanimité pour quatre autres exemples $(376 \mathrm{a} 16,18,31 ; 376 \mathrm{~b} 1)$ dans un passage où les copistes ont manifestement été déroutés par les

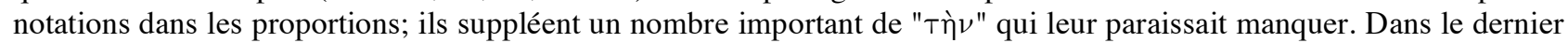
exemple du passage, $376 \mathrm{~b} 7$ il y a encore divergence, mais cette fois c'est la proportion toute entière qui manque dans certains mss.
} 
Quoi qu'il en soit, si cette notation n'est pas la formule "archaïque", ce n'est pas non plus la formule simple que l'on rapporte d'habitude aux mathématiciens hellénistiques ${ }^{43}$.

5. Enfin et surtout, il se pourrait que la formule complexe n'ait pas été utilisée pour l'ensemble d'une démonstration, mais seulement dans les parties que l'on appelle ecthèse (détermination) et

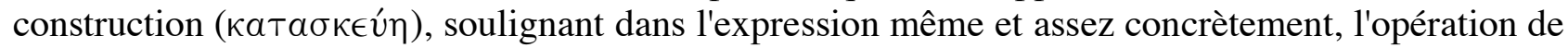
mise en place du lettrage sur un schéma et le baptême des objets qui lui sont ainsi associés ou qui sont construits au fur et à mesure ${ }^{44}$. Le critère serait alors discriminant seulement pour les portions correspondantes. Par exemple, dans la séquence mentionnée ci-dessus : «'́ $\phi$ ' îs Tà $\mathrm{N} M$ 》 (15) est un tel baptême; «ámò Tôv H K » (12), « Tò $\mathrm{M}$ » (14) n'en sont pas et des formes simplifiées pourraient être alors tolérées; quant à « Tô $\epsilon \phi$ ' nouveau baptême, la lettre "A" pouvant désigner successivement le plan de l'horizon, le grand cercle intersection de celui-ci et de la sphère "météorologique" et, maintenant, l'un de ses deux demi-cercles 45 . Le fait qu'il s'agit maintenant d'un demi-cercle seulement a son importance dans la formulation aristotélicienne d'unicité pour le point $\mathrm{M}$ (afin d'exclure son symétrique par rapport à la droite HK).

Si cette hypothèse s'avérait exacte, nous restreindrions davantage encore la portée du premier critère de Tannery, mais peut-être en augmenterions-nous la pertinence. Pour tenter de valider cette hypothèse il convient d'examiner les habitudes du Stagirite en la matière, ce qui nous contraint à faire un certain détour.

\section{B. Les arguments aristotéliciens de style mathématique}

1. Aristote utilise assez souvent des arguments comportant un lettrage afin de désigner de manière abrégée et abstraite certaines entités. Les exemples les plus célèbres se trouvent dans les traités logiques pour la formalisation des syllogismes. Mais dans ses traités physiques il utilise également une catégorie d'arguments que j'appelle « de style mathématique », dans lesquels des lettres sont utilisées pour désigner des grandeurs, des temps, des "densités", des mouvements ... Pour les distinguer des arguments de type logique on peut remarquer qu'ils comportent fréquemment un énoncé général, suivi d'une exemplification, souvent introduite par des formules comme « É $\sigma T \omega$

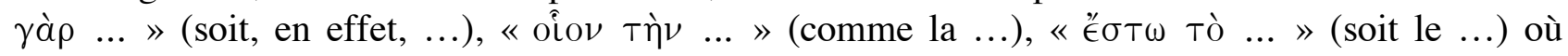
intervient un lettrage correspondant très certainement à l'usage d'un diagramme. Suit une portion démonstrative où l'on "intervient" sur les entités désignées par des lettres, elle-même suivie de la répétition, en conclusion, de l'assertion générale initiale ${ }^{46}$.

2. Cette structure n'est pas sans rappeler celle de la Proposition euclidienne avec ses divisions très formelles dont Proclus détaille la liste et les significations : énoncé ( $\pi \rho o ́ T a \sigma \iota s)$, exemplification

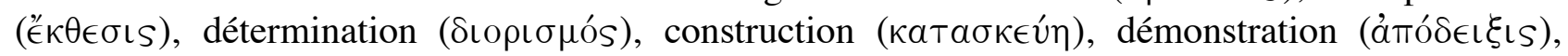

\footnotetext{
43 Dans son analyse, Knorr relève aussi la notation complexe "archaïque" et la considère comme un indice d'ancienneté. Puisqu'à la différence de Heath il n'admet pas qu'Aristote soit l'auteur de cette démonstration, le critère lui sert à garantir l'ancienneté relative du passage et de son hypothétique auteur aristotélicien. En revanche il n'a pas relevé la notation intermédiaire. Pour d'autres occurrences de cette dernière dans le corpus aristotélicien, v. infra, B, § 4.

${ }^{44}$ Cf. infra, B, $\$ 5,7-8$.

45 Cette variabilité apparente dans la signification du lettrage n'a d'ailleurs aucune importance, contrairement à ce que suggère Tannery. La fonction des lettres sur un diagramme géométrique grec est visuelle et non pas symbolique. Il ne s'agit pas de variables en correspondance biunivoque avec un objet mathématique qu'elles représenteraient dans le discours, mais de "déictiques" et "A" peut faire voir une demie sphère, un cercle, ou un demi-cercle sur le même diagramme. Ce phénomène de multi-désignation est fréquent dans les Éléments d'Euclide où les mêmes lettres peuvent désigner à la fois un cercle et un polygone régulier, inscrit dans ledit cercle. Les travaux récents de R. Netz sur les diagrammes sont très clairs quant à la valeur iconique des lettrages (v. [Netz, 1999]).

46 Cette division est lisible dans les trois exemples que contiennent les Météorologiques, à savoir l'analyse géométrique du halo (III, 373 a6-19) et bien entendu celle de l'arc-en-ciel, en deux cas de figures, objet de la présente discussion.
} 


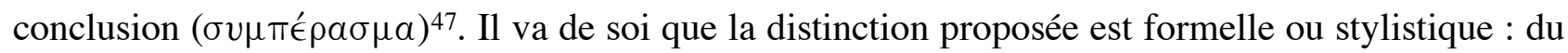
point de vue des contenus, Aristote parle pour l'essentiel de philosophie naturelle, pas de mathématiques. En utilisant cette caractérisation, j'ai déterminé un échantillon de près d'une quarantaine d'« arguments de style mathématique » (en abrégé ASM) dans l'œuvre du Stagirite ${ }^{48}$. La signification de ce nombre "quarante" ne doit pas être majorée; il s'agit d'un ordre de grandeur car il y a une certaine part d'arbitraire dans les découpages que j'ai opérés. Ainsi j'ai compté l'analyse de l'arc-en-ciel pour deux ASM correspondant aux deux cas de figure envisagés (astre au levant ou au couchant; astre au-dessus de l'horizon); on pourrait n'en compter qu'un seul ou au contraire trois (en distinguant en outre la partie E. «Applications à l'apparition de l'arc-en-ciel »= 58-63).

3. Ces arguments sont principalement contenus dans le De calo $\left(8 \mathrm{ASM}^{49}\right)$ et surtout la Physique $(27 \mathrm{ASM})^{50}$. Leur répartition, dans les différents Livres de ce traité, est très inégale : aucun dans les Livres I-III (ceci n'a rien d'étonnant dans la mesure où Aristote y traite des concepts les plus généraux de cette science ${ }^{51}$; un seul ASM dans le Livre IV $^{52}$ et aucun dans le Livre V. Mais j'ai compté 20 ASM dans le Livre VI ${ }^{53}, 3$ dans le Livre VII ${ }^{54}, 3$ dans le Livre VIII ${ }^{55}$. La différence entre les Livres I-V d'une part et les Livres VI-VIII d'autre part est donc claire : à une exception près, tous les ASM appartiennent à l'hypothétique $\Pi \epsilon \rho i$ $\kappa \iota \nu \eta ́ \sigma \epsilon \omega S$ (L. VI-VIII). À l'intérieur du second groupe, le Livre VI est véritablement le plus "mathématique" des Livres de la Physique d'Aristote; les 26 arguments des Livres VI-VIII interviennent tous dans la discussion du changement et du mouvement et dans l'analyse de ses propriétés.

Pour conclure cet aparté on peut aussi remarquer que sur les 38 ASM de l'ensemble Physique-Météorologiques- De Caelo, les démonstrations directes pour soutenir une thèse positive sont seulement une dizaine ${ }^{56}$ et donc que trois quarts des ASM font donc appel à une démonstration indirecte et ont, de ce fait, une coloration polémique. Les démonstrations d'impossibilité et autres argumentations qui visent à réfuter - le mouvement est impossible dans le vide; il n'y a pas de grandeurs sans parties; il n'y a pas de mouvement dans l'instant; il n'existe pas de corps infini ayant un poids fini ... - représentent d'ailleurs près de la moitié de l'échantillon (16 ASM sur 38). C'est dire la force de réfutation qu'Aristote reconnaît à ce genre d'argumentation. Cet

47 V. [Pr., in. Eucl. I], p. 203, 1. 1-5.

48 Cet inventaire fait partie d'un travail en cours sur l'usage des mathématiques chez Aristote qui a fait l'objet d'une présentation dans le Séminaire sur la philosophie naturelle des Grecs animé par P. Pellegrin et A. Laks ainsi que dans le Séminaire 'Aristote' de la Maison Française d'Oxford (1995), animé par D. Charles, M. Frede, D. Furley, E. Hussey et P. Pellegrin. J'en réserve les détails à une publication ultérieure.

${ }^{49}$ L. I, Ch. 5, 271 b26-272 a 20; 272 b 25-28 (Deux preuves de ce que le corps mû circulairement est limité); Ch. 6, 273 a 27-b29 (Il n'existe pas de corps infini ayant un poids fini); Ch. 7, 274 b33-275 a24 (Impossibilité que l'infini pâtisse sous l'action de - ou exerce une action sur - un être limité); 275 a24-b4 (Impossibilité que l'infini pâtisse sous l'action d'un infini). L'ensemble de ces arguments débouche sur le corollaire qu'il n'y a pas de corps infini. L. II , Ch. 4, 287 b414 (Sphéricité de la surface de l'eau). L. III, Ch. 2, 301 a26-b1 (Tout corps a pesanteur ou légèreté); 301 b1-17 (Tout corps sans pesanteur ni légèreté est mû par contrainte).

50 Ils sont absents de la Métaphysique, de la Politique, de la Poétique, de la Rhétorique..., du De anima et du De Generatione et corruptione, mais on en trouve un dans l'Éthique à Nicomaque (V, 7, 1132 a35-b 12). En V, 8, 1133 a612 et 1133 b 22-25 il s'agit seulement de désignations à l'aide de lettres sans intervention ultérieure sur les entités désignées, comme dans ce que j'appelle les arguments logiques.

51 Cela dit, dans le L. III il avait l'opportunité d'utiliser des ASM pour l'analyse de l'infini, comme dans le De calo, mais il n'en fait rien.

52 Ch. 8, 215 a29-216 a11.

53 Ch. 1, 231 b 18-232 a 20. Ch. 2, 232 a27-b5; 232 b5-14; 232 b26-233 a5; 233 a31-b15; 233 b15-32. Ch. 3, 234 a2631. Ch. 4, 234 b21-235 a10; 235 a13-37. Ch. 5, 235 b32-236 a6; 236 a13-27; 236 a27-35; 236 b10-18. Ch. 6, 236 b2532; 236 b32-237 a11; 237 a30-237 b6. Ch. 7, 237 b26-238 a17; 238 a36-b12. Ch. 9, 239 b33-240 a18. Ch. 10,240 b17241 a6.

54 Ch. 1, 242 a4 -15. Ch. 4, 248 a18-b6 (argument de type métamathématique sur l'incomparabilité du rectiligne et du circulaire). Ch. 5, 249 b30 -250 a 19.

${ }^{55}$ Ch. 8, 262 b 8-21. Ch. 10, 266 a12-23; 266 a24-b 26.

${ }^{56}$ Mais certaines sont très importantes : le temps est continu (Phys., VI, Ch. 2, 232 b26-233 a5); le temps et le domaine du mouvement ont les mêmes divisions (Phys., VI, Ch. 4, 235 a13-37), sans oublier l'analyse de la forme du halo. 
échantillon nous fournit un terme de comparaison d'une taille raisonnable quant aux habitudes du Stagirite en matière de désignation des lettrages et de formulation des proportions.

4. Commençons avec la question des lettrages. Près de $90 \%$ de nos ASM contiennent deux systèmes de désignation, dans la plupart des cas : "complexe" / "simple" (25 ASM), mais aussi : "intermédiaire" / "simple" (2 ASM), voire les trois systèmes ("complexe" / "intermédiaire" /

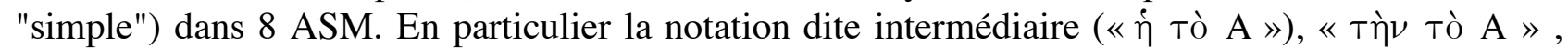
«ं тò $\mathrm{AB} \Gamma$ »...) n'est pas seulement présente dans nos loci du Livre III des Météorologiques ${ }^{57}$, mais elle est aussi attestée dans la Physique ${ }^{58}$ et le De calo ${ }^{59}$.

Si l'on classe les ASM en fonction du nombre de désignations d'objet ${ }^{60}$, pour tenir compte de la complexité formelle et de la longueur de l'argument, et que l'on note le nombre de systèmes de notations utilisés, on obtient le tableau suivant :

\begin{tabular}{|c|c|c|c|c|c|c|c|c|c|c|c|c|c|}
\hline $\begin{array}{c}\text { Nombre de } \\
\text { Désignations }\end{array}$ & 136 & 38 & 33 & 27 & 26 & 22 & 21 & 20 & 19 & 16 & 14 & 13 & 12 \\
\hline $\begin{array}{c}\text { Nombre de } \\
\text { syst. de not. }\end{array}$ & 3 & 3 & 3 & 3 & $3 / 261$ & 2 & 2 & $3 / 2$ & 2 & 2 & 3 & $2 / 2 / 2$ & 2 \\
\hline \\
$\qquad$\begin{tabular}{c}
9 \\
\hline
\end{tabular} \\
\hline \\
\hline
\end{tabular}

Très clairement le nombre de systèmes de notation est fortement lié au nombre des désignations. Ceci s'explique parfaitement lorsqu'on examine la manière dont sont utilisés lesdits systèmes, en particulier dans les ASM de la Physique et du De calo : le recours à 2 ou 3 systèmes de notations est généralement fonctionnel, et souvent même doublement fonctionnel.

5. Une première fonction consiste à utiliser la notation complexe (parfois l'intermédiaire) pour la seule première occurrence (les premières quand il y en a beaucoup) d'une ou plusieurs entités, la notation simple pour les suivantes ${ }^{62}$. C'est ce qu'on peut appeler la fonction de «baptême » des notations non simples. Parfois la notation complexe n'apparaît que pour la première occurrence du premier objet désigné; il n'y a alors qu'une seule notation complexe dans l'ensemble de l'argument, la première et il s'agit simplement d'initialiser le lettrage du diagramme ${ }^{63}$.

La seconde fonction réside dans le fait que l'utilisation de deux systèmes distincts se conforme souvent à une opposition de nature entre objets considérés (en dépit de quelques exceptions), par exemple un système étant utilisé pour les grandeurs, un autre pour les temps, les poids ... ou encore un pour des totalités, l'autre pour des parties ${ }^{64} \ldots$ Les trois ASM des

57 On trouve deux autres occurrences de cette formule dans la partie « application à l'apparition de l'arc-en-ciel » en 377 a22-23. Elle se trouve également dans l'analyse géométrique du halo (4 occurrences : III. 3, 373 a 2, 12, 12-13, 13)

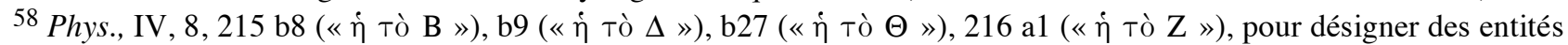
préalablement introduites comme des grandeurs $(\mathrm{B}, \Delta)$ ou comme un corps $(\mathrm{Z})$, donc des choses dont le nom grec est neutre et non féminin! Phys., VI, 1, 231 b 27, b28; 232 a1, a22 [il s'agit de grandeurs ou de longueurs (féminin)]; Phys., VII, 5, 250 a8, a12, a13 [il s'agit d'une puissance, d'une longueur (féminin), mais aussi de l'objet mû (neutre)]; Phys., VIII, 10, 266 a 19 (il s'agit d'un temps (masculin) désigné par « ò Tò Z » (texte de l'édition Ross).

${ }^{59}$ De calo, I, 5, 272 a14, 15, 18, 19, 22, b25, 26 (il s'agit de lignes).

60 Autrement dit en comptant les lettres ou groupes de lettres; notre échantillon en contient près de 500 mais il faut noter que les deux ASM du ch. III. 5 des Météorologiques en représentent à eux seuls près du tiers !

61 Il y a 2 ASM comportant 26 désignations d'objets, l'un avec les 3 systèmes de notation; l'autre avec deux, d'où notre notation $3 / 2$. Je fais une convention similaire à chaque fois.

62 V. par exemple Phys., VI, 1, 231 b 18-232 a 20; VI, 2, 233 a31-b15; VI, 9, 239 b33-240 a18. De calo, I, 7, 275 a24b4.

${ }^{63}$ V. par exemple Phys., VI, 3, 234 a26-31; VI, 5, 236 a13-27; VI, 5, 236 b10-18, VI, 6, 236 b25-32; VI, 7, 237 b26-238 a17; 238 a36-b12; VIII, 8, 262 b 8-21. Meteor., III. 3, 373 a3-19.

64 V. par exemple Phys., VI, 10, 240 b17-241 a6 [mobile (not. simple) / temps (not. complexe)]; De caelo, I, 5, 271 b26272 a 20; 272 b 25-28 [droite (not. complexe ou intermédiaire) / point (not. simple)]; I, 6, 273 a 22-b29 [poids (not. complexe)] / grandeur (not. simple)]; II, 4, 287 b4-14 [rayons (not. simple) / autres éléments géométriques (not. complexe)]; III, 2, 2, 301 a26-b1 et 301 b1-17 [corps (not. complexe)] / grandeur (not. simple)] ... En fait plusieurs ASM combinent les deux fonctions que j'ai distinguées. 
Météorologiques sont purement géométriques et offrent donc moins de possibilités que les arguments de la Physique ou du De calo pour la manifestation de cette seconde fonctionnalité. Mais relevons toutefois que dans l'analyse géométrique du halo (III. 3, 373 a3-19) la notation intermédiaire est réservée à certaines catégories particulières de lignes : les trois lignes brisées : $\mathrm{A} \Gamma \mathrm{B}, \mathrm{AZB}, \mathrm{A} \Delta \mathrm{B}(373 \mathrm{a} 7-8)$ et les trois perpendiculaires qui leur sont associées : ГE, ZE, $\Delta \mathrm{E}(373$ a 12-13), alors que les autres droites sont désignées grâce à la notation simple.

6. À partir de ces sommaires considérations de type statistique il apparaît clairement que l'on ne peut généralement pas appliquer le critère de Tannery aux ASM en question - et, par conséquent, extirper les portions de texte où figurent très majoritairement les notations simples - puisque, dans la plupart des cas, cela reviendrait à faire disparaître entièrement certaines catégories d'objets (celles désignées par les notations simples) d'arguments dans lesquels ils jouent pourtant un rôle essentiel. Il me semble possible de procéder comme le fait Tannery dans le cas du chapitre III. 5 des Météorologiques seulement dans 7 ou $8 \mathrm{ASM}^{65}$. Dans quelques cas cela reviendra à considérer une partie de la portion de style mathématique, voire la totalité, comme inauthentique; ainsi dans

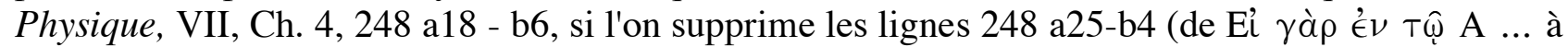

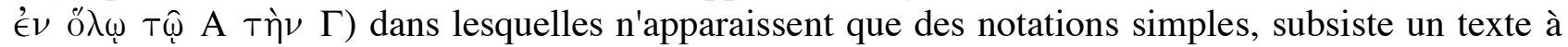
peu près cohérent. Mais, pour la trentaine d'ASM restants, les plus longs et les plus significatifs, c'est impossible. Il n'y a donc que deux possibilités :

- Soit accepter ces textes (à peu près) tel qu'ils sont;

- Soit supposer qu'ils ont été simplifiés (partiellement) à partir d'un état comportant seulement des notations complexes par des réviseurs, éditeurs et/ou copistes, non pas de manière aléatoire, mais selon des principes relativement constants et rationnels. Mais cette hypothèse supplémentaire, outre qu'elle est passablement ad hoc, ne simplifie pas vraiment notre affaire puisqu'une telle simplification aurait également pu être appliquée au chapitre III. 5 des Météorologiques.

7. Quoi qu'il en soit, la pertinence de la première fonctionnalité ("baptême") pour la notation complexe "archaïque" est confirmée par les exemples géométriques conservés (ou probables). Car, contrairement à ce que dit Tannery, cette notation n'est pas seulement attestée chez Aristote et dans le fragment d'Hippocrate-Eudème. On la trouve également chez des auteurs comme Archimède ${ }^{66}$, Philon de Byzance ${ }^{67}$ (fin du III $^{\mathrm{e}}$ s. avant notre ère) et peut-être Apollonius de Perge ${ }^{68}$. Pour ce dernier auteur en effet, il ne faut pas oublier que nous ne disposons pas du texte grec original des Coniques, mais seulement de la réédition des quatre premiers Livres par Eutocius d'Ascalon (VI ${ }^{\mathrm{e}} \mathrm{s}$. de notre ère), sans doute modernisée au niveau des notations. Mais 25 occurrences de la notation complexe subsistent dans le fragment du Livre II de la Collection mathématique de Pappus qui suit explicitement un exposé d'Apollonius ${ }^{69}$. Il est à craindre que le cas des Éléments d'Euclide -

65 Phys., VI, Ch. 2, 233 a31-b15; Ch. 4, 235 a13-37; Ch. 5, 235 b32-236 a6; Ch. 6, 236 b32-237 a11; Ch. 6, 237 a30237 b6; Ch. 7, 238 a36-b12. Phys., VII, Ch. 4, 248 a18-b6).

66 V. Conö̈des et Sphérö̈des, Prop. 2, [Archimedis opera ..., 1972], t. I, p. 266, 1. 17; 1. 22; p. 268, 1. 19-20; Prop. 3, Ibid., p. 272, 1. 18; Prop. 4, Ibid., p. 276, 1. 7, 8, 9; Prop. 19, Ibid., p. 338, 1. 5, 6, 8-9, 10-11; Prop. 25, Ibid., p. 376, 1. 3, 12; Prop. 27, Ibid., p. 396, 1. 12; Prop. 29, Ibid., 1. 414, 1. 5. Spirales, Prop. 12, [Archimedis opera ..., 1972], t. II, p. 46, 1. 27; Prop. 13, Ibid., p. 48, 1. 20; Prop. 15, Ibid., p. 52, 1. 22; Prop. 16, Ibid., p. 56, 1. 1; Prop. 20, Ibid., p. 72, 1. 18; Prop. 21, Ibid., p. 76, 1. 21-22; Prop. 22, Ibid., p. 80, 1. 24; Prop. 23, Ibid., p. 84, 1. 58; Prop. 24, Ibid., p. 86, 1. 11; Prop. 25, Ibid., p. 92, 1. 24; Prop. 26, Ibid., p. 102, 1. 13; Prop. 28, Ibid., p. 116, 1. 22. Corps flottants, Prop. II, 8, Ibid., p. 374, 1. 11. Que la notation complexe soit encore utilisée par Archimède a été signalé dans [Federspiel, 1992], p. 24. Il signale également une expression du même genre mais avec la préposition "É $\nu$ ", utilisée très souvent par Archimède quand il s'agit de lettrage concernant des solides. Ajoutons qu'il est sans doute significatif que ces occurrences apparaissent dans les traités maintenus dans l'original dorien, et non dans ceux convertis en langue commune comme Sphère et cylindre ou Mesure du cercle.

67 V. [Philon, Belopoeica ..., 1971], p. 110, § 52, 1. 7-8, 12, 15, 16; p. 128, §63, 1. 19, 20-21, 24, 29; p. 130, § 64, 1. 3-4, $12,13,17,18,19 ;$ p. 130, $\S 65,1.7-8,11-12$; p. 132, §66, 1. 4; p. 144, § 73, 1. 2; p. 148, § 75, 1. 30-31. Que la notation complexe soit encore utilisée par Philon a été signalé dans [Knorr, 1986], n. 28, pp. 141-142.

68 V. [Apollonii Pergaei ..., 1974], t. I, Prop. II. 46, p. 266, 1. 16; p. 268, 1. 1; Prop. III. 13, p. 338, 1. 12-13.

${ }^{69}$ V. [Papp., Coll. math.] L. II, $\S \S$ XV, XVII, p. 2, 1. 14-15; p. 4, 1. 1, 2, 4, 5, 9, 16; p. 6, 1. 7, 9, 10-11, 12, 12-13, 13-14, 14, 17, 18, 20, 25, 26, 28; p. 8, 1. 1, 3, 7. Le fait est signalé aussi dans [Federspiel, 1992], p. 24, en part. n. 26. Quand il 
souvent pris comme terme de comparaison, en particulier par Tannery et Heath - soit du même genre. En effet, non seulement le traité d'Euclide a été réédité au IVe $\mathrm{s}$. de notre ère, mais le texte a subi des ajouts et des remaniements d'une manière continue, lesquels pourraient bien avoir produit l'état simplifié des lettrages que nous observons dans le texte transmis.

8. Si, comme nous avons essayé de le montrer dans un précédent travail ${ }^{70}$, on admet la pertinence des versions médiévales, en particulier des traductions arabo-latines attribuées à Adélard de Bath et à Gérard de Crémone ${ }^{71}$, pour obtenir des informations sur un (ou des) état(s) antérieur(s) du texte grec, on observe facilement, surtout chez Gérard, le phénomène suivant : en général le lettrage est simple, par exemple « circulus gdb » pour désigner le cercle GDB; mais dans un certain nombre de cas, la formulation se complique : « circumducatur circulus, super quem sunt $g d \boldsymbol{b}$ » (que soit décrit un cercle, sur lequel sont $G, D, B » 72$. Je crois que l'expression « super quem sunt $\boldsymbol{g} \boldsymbol{d} \boldsymbol{b} »$ restitue

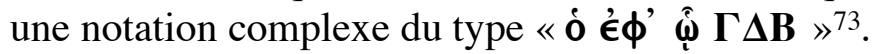

Sans prétendre à l'exhaustivité, j'ai relevé une soixantaine d'occurrences de ce type dans la version de Gérard, une vingtaine dans celle d'Adélard ${ }^{74}$, très inégalement réparties : elles sont très rares dans les Livres arithmétiques ${ }^{75}$ et le Livre $\mathrm{X}^{76}$, absentes du Livre XI, mais on compte quand même une bonne vingtaine dans le Livre I et une dans chacune des ecthèses des Propositions 1 à 9 du Livre II. Ce qui importe, c'est que pratiquement toutes ces formulations se trouvent dans la partie ecthèse-détermination correspondant à l'initialisation du lettrage, rarement dans la portion "construction" (kataskeuê), et pas dans la démonstration proprement dite. Or une localisation du même genre s'observe dans la plupart des exemples archimédiens ${ }^{77}$, conservés en grec cette fois.

9. Pour ma part je suis donc convaincu que les formules latines en question sont les vestiges de notations complexes que portai(en)t le(s) copie(s) grecque(s) utilisées par les traducteurs arabes et sans doute aussi par le texte hellénistique des Éléments, même si ceci est impossible à prouver ${ }^{78}$. Je crois aussi que l'adoption systématique des notations simples, y compris dans les ecthèses, s'est faite à une époque nettement postérieure à ce que croit Tannery, autrement dit que l'usage de notations complexes dans les ecthèses était encore chose commune chez les mathématiciens de la première période de l'École d'Alexandrie (300-150 avant notre ère environ, ou, si l'on préfère, d'Euclide à Hypsiclès $)^{79}$.

affirme que la notation complexe n'était pas utilisée dans les Coniques (ibid. ), il semble avoir perdu de vue l'origine eutocienne du texte grec qui nous est parvenu.

70 V. [Rommevaux, S., Djebbar, A., Vitrac, B., 2001].

${ }^{71}$ Dans ce qui suit j'utilise l'abréviation [Gérard Cr., Busard, 1984].

72 Cet exemple est emprunté à la Prop. I. 1 (v. [Gérard Cr., Busard, 1984], p. 3, 1. 58-59).

${ }^{73}$ Il ne s'agit pas de préciser qu'un cercle est défini par trois de ses points (c'est l'interprétation que l'on peut donner des deux exemples apolloniens mentionnés ci-dessus, n. 68. Cf. [Federspiel, 1992], p. 24) puisque la formule s'utilise pour des droites désignées par une seule lettre. V. par ex. Prop. II. 1, [Gérard Cr., Busard, 1984], p. 39, 1. 45.

${ }^{74}$ Celui-ci utilise "supra" et non "super"; dans la plupart des cas il omet le verbe "être". Une dizaine d'occurrences sont communes à Adélard et à Gérard.

75 On en trouve seulement deux, appliquées à des nombres, dans les Propositions VIII. 11-12 dans [Gérard Cr., Busard, 1984], p. 197, 1. 45-46 et p. 198, 1. 25-26. On ne les trouve pas dans la version d'Adélard dont le texte, pour ces deux théorèmes, est au demeurant passablement différent.

76 [Gérard Cr., Busard, 1984], Prop. X. 13, p. 243, 1. 58-59; X. 24, p. 251, 1. 55-56; X. 85, p. 307, 1. 25; X. 86, p. 308 , 1. 40; X. 87, p. 309, 1. 26-27; X. 88, p. 310, 1. 10.

77 Exceptions : Conö̈des et Sphérö̈des, Prop. 2, [Archimedis opera ..., 1972], t. I, p. 268, 1. 19-20; Prop. 19, Ibid., p. 338, 1. 5, 6, 8-9, 10-11; Corps flottants, Prop. II. 8, [Archimedis opera ..., 1972], t. II, p. 374, 1. 11. Les exemples dans le traité des Spirales (v. supra, n. 66) sont particulièrement éclairants.

${ }^{78}$ Les quelques fragments de papyri euclidiens qui nous sont parvenus ne nous sont d'aucun secours : deux portent sur des définitions; un troisième est sans lettrage. Quant au Pap. Fayoum 9 qui contient une bonne partie de la démonstration de I. 39, il n'inclut malheureusement pas la partie de l'ecthèse correspondant à l'initialisation du lettrage. V. [Euclidis Elementa ..., 1969], p. 188, 1. 4.

${ }^{79}$ Par conséquent je ne partage pas l'avis de Knorr qui, du fait que Philon continue à utiliser la formule complexe de lettrage, en déduit que celui-ci ne devait pas être en contact avec les chercheurs les plus avancés du temps en géométrie (v. [Knorr, 1986], n. 28, pp. 141-142). Je ne crois pas non plus qu'il faille voir dans le même usage, de la part 
10. Quant aux manières d'exprimer les proportions, le dossier est plus mince : 9 ASM seulement ${ }^{80}$ sont concernés même si les moyens utilisés sont assez variés. Dans la mesure où c'est la formule idiosyncrasique en "öтє $\rho$ " attestée en Météorologiques, III. 5 qui motive cette investigation, il faut donc privilégier les expressions générales ${ }^{81}$ de la proportionnalité et, plus précisément, les formules à 4 places (au moins) du type : (i) « ce que A est relativement à $\mathrm{B}, \mathrm{C}$ l'est relativement à $\mathrm{D}$ » (öt $\epsilon \rho$

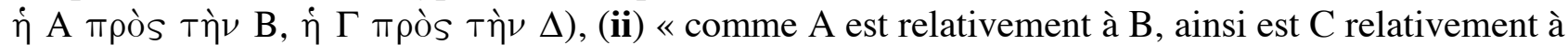

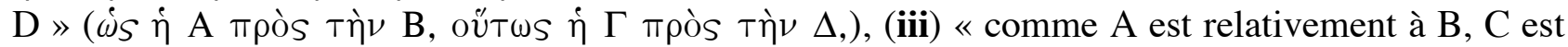

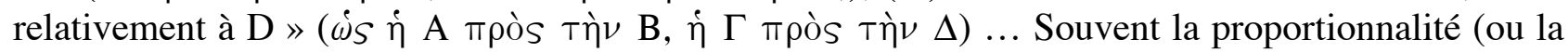
non proportionnalité) est signifiée autrement, à l'aide d'expressions dans lesquelles apparaissent des

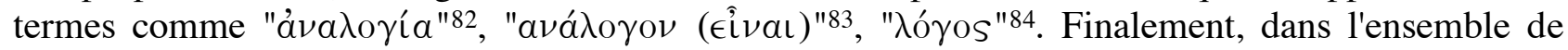
notre échantillon (y compris les deux témoignages supplémentaires que j'adjoins aux ASM), nous trouvons seulement 22 formules lettrées à quatre places (au moins) dont près de la moitié (10) se trouve dans le chapitre III. 5 des Météorologiques.

Autre constat facile à faire : alors qu'il y a 8 occurrences de la formule en "ö $\pi \epsilon \rho$ " (i) dans celui-ci, aucune ne se trouve dans les huit autres ASM (4 dans la Physique ${ }^{85}, 4$ dans le De calo ${ }^{86}$ ). Cela dit, contrairement à ce que suggère Tannery, on n'y trouve pas non plus la formule

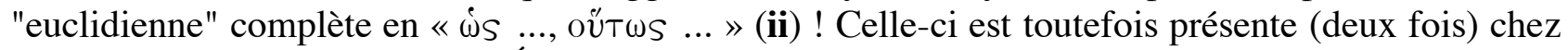
Aristote, dans le passage de l'Éthique à Nicomaque, mais il s'agit alors d'une quasi-citation du théorème dit de permutation (cf. Eucl., Él. V. 16) ${ }^{87}$. Un peu plus loin (1131 b14-15) on lit d'ailleurs

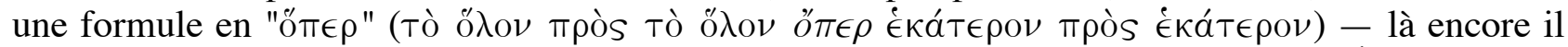
s'agit de renvoyer à un célèbre résultat de la théorie des proportions (cf. Eucl., Él. V. 12) occurrence qui ne contredit cependant pas ce qui précède dans la mesure où il s'agit d'une expression dénuée de lettrage. De fait Aristote utilise le plus souvent la formule "euclidienne" abrégée, avec seulement "is" (iii) ${ }^{88}$ et, rarement, une expression formée à l'aide du pronom relatif simple "o" "89 que l'on trouvait déjà chez Platon" 90.

d'Archimède, une confirmation du fait que celui-ci utilisait des sources préeuclidiennes, comme le suggère [Federspiel, 1992], p. 24, alors que, comme Knorr lui-même l'a admis, dans le traité des Spirales, Archimède se réfère à la théorie des proportions du L. V des Éléments tout en y utilisant la formule complexe de lettrage.

80 On peut leur adjoindre deux autres célèbres passages : De calo, I, 6, 273 b29-274 a18; Eth. Nic., V, 6-7, 1131 a29b17, lesquels, s'ils ne sont pas des ASM à proprement parler, donnent de précieuses indications sur les formulations aristotéliciennes de la proportionnalité.

81 Même quand une relation générale de proportionnalité vaut entre quatre grandeurs, Aristote peut l'énoncer sur un cas particulier - souvent le rapport double ou moitié - dont le lecteur est censé percevoir le caractère générique. V. par ex. Phys., IV. 8, 215 b8-10; VII. 5, 250 a3-4; 6-7.

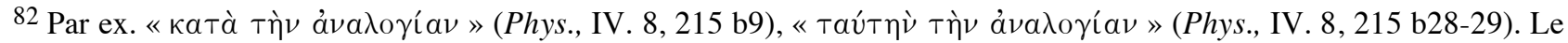
terme apparaît aussi dans De caelo, I, 6, 273 b32-274 a2 et dans Eth. Nic., V, 6-7, 1131 a31, b15; en 1131 b12

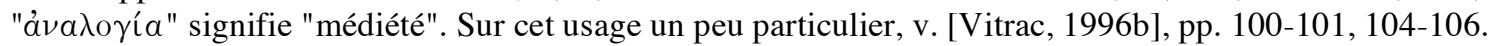

${ }^{83}$ V. par ex. Phys., IV. 8, 216 a7; VII. 5, 250 a4, a9; De calo, I, 6, 273 b3; 274 a5-6; I. 7, 275 a9, b1. Eth. Nic., V, 6-7,

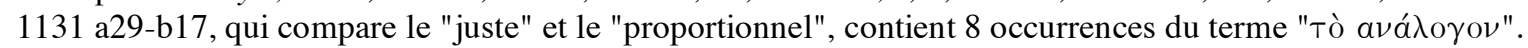

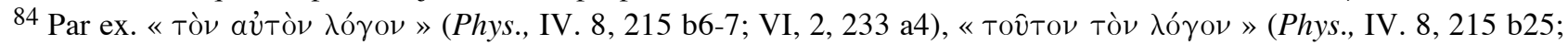

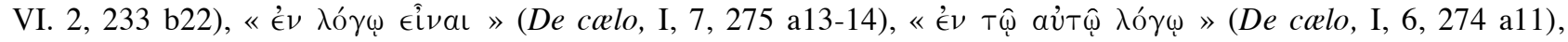

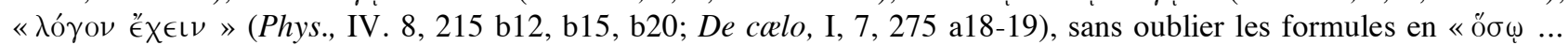
тобоútழ » (Phys., IV. 8, 215 b4-5; 215 b31-216 a1).

85 Phys., IV. 8, 215 a29-216 a11; VI, 2, 232 b26-233 a5; VI, 2, 233 b15-32; VII, 5, 249 b30-250 a19.

${ }^{86}$ De calo, I, 6, 273 a22-b29; I, 7, 274 b33-275 a24; I, 7, 275 a24-b4; III, 2, 301 b1-17.

871131 b5-7.

88 Phys., IV. 8, 216 a7; VII, 5, 250 a15; De calo, I, 6, 273 b1-2; I, 6, b19-20; III, 2, 301 b11-13; Meteor., III, 5, 376 a10-11; Eth. Nic., V, 6-7, 1131 b6-8. Cette formule est d'ailleurs la plus fréquemment utilisée dans la citation d'Apollonius par Eutocius (18 occurrences) alors que la formule euclidienne complète (ii) n'est attestée que trois fois (consécutives) dans la deuxième partie de la preuve (p. 184, 1. 10-11, 11-13, 13-14), sans doute altérée (v. Annexe III).

${ }^{89}$ De calo, I, 7, 275 a6; a17-18; a30.

90 Gorgias, 465 c1-5. Dans le Timée, l'expression de la proportionnalité est encore plus simple, sans adverbe ni pronom relatif; (32 a1-3) : « ... ce que le premier est à lui-même, lui-même l'est au dernier; et que, inversement aussi, ce que le

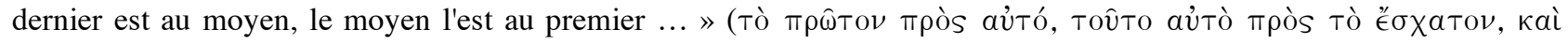




\section{Les reconstructions possibles du texte aristotélicien du premier cas de figure}

1. En résumé les critères philologiques proposés par Tannery cherchaient à identifier les interpolations ajoutées au traitement géométrique aristotélicien de l'arc-en-ciel : de petites incises et corruptions ponctuelles dans la première partie (7-16), la substitution d'une explication alternative à la fin du premier cas de figure (43-47) et surtout l'intercalation centrale (18-39) d'une preuve accessoire.

J'ai essayé de montrer les incertitudes qui entourent l'utilisation du premier critère concernant les différents systèmes de lettrage que porte le texte. Cela conduit à remettre en cause ou du moins à relativiser les conclusions de Tannery concernant le début et la fin du chapitre, lesquelles reposent sur ce seul critère ${ }^{91}$. En revanche le second critère, concernant l'expression de la proportionnalité, malgré sa portée limitée, m'a paru mieux fondé : l'idiosyncrasique formulation en "ö $\epsilon \epsilon \rho$ " (i) n'est le résultat ni de l'inadvertance des copistes, ni d'une corruption tardive : Alexandre cite la proportion contenue dans les lignes 376 a25-26 (cf. 30) de cette manière pour souligner qu'elle inverse l'ordre habituel des termes ${ }^{92}$. En revanche il ne dit rien de la notation intermédiaire que l'on trouve également à cet endroit. À ce témoignage contrasté d'Alexandre s'ajoute l'inventaire aristotélicien négatif rapporté dans le paragraphe qui précède.

2. Il ne s'agit là que d'indices, à eux seuls non décisifs, mais confortés par d'autres informations :

- L'objectif explicitement énoncé par Aristote au début du chapitre n'inclut pas vraiment la démonstration de la forme circulaire de l'arc-en-ciel. L'accent est mis sur la taille de la portion de cercle qu'il représente (ainsi que sa variation).

- La consultation d'un diagramme géométrique est censée convaincre le lecteur d'une manière évidente et donc sans doute assez rapide. C'est effectivement ce que l'on observe dans le traitement $\mathrm{du}$ second cas de figure et dans les applications au moment d'apparition de l'arc-en-ciel, mais certainement pas dans l'exposé embrouillé du premier cas de figure si l'on en maintient intégralement le texte.

- Ajoutons que la comparaison avec d'autres passages de style mathématique du corpus aristotélicien ne livre rien de semblable.

Il est donc hautement probable, comme l'affirme également Madame Merker ${ }^{93}$, que notre texte a été altéré, en fait fortement "enrichi". Le second critère de Tannery jette le doute sur la portion (21-39) dans laquelle on trouve les formulations suspectes en "ő" $\pi \epsilon \rho$ " et, pour conserver une cohérence minimale au texte, il faut lui adjoindre l'ecthèse et le début de la construction des sections (18-20). Jusque-là notre conclusion est la même que celle de Tannery, même si les arguments ne sont pas tout à fait les mêmes. Mais il demeure toute une série de problèmes : a. Où commence l'interpolation centrale ? b. Où finit-elle ? c. Ses bornes étant fixées, quel est exactement son contenu? Les réponses à ces trois questions, fondamentales pour notre propos, ne sont pas indépendantes.

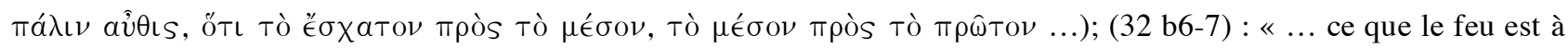

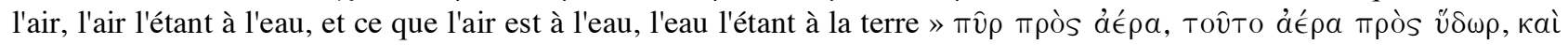

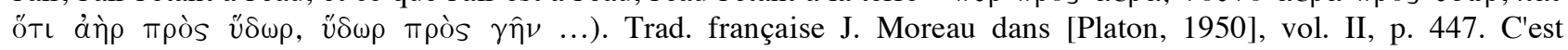
également de cette manière qu'Archytas définit la médiété géométrique : « [Il y a moyenne] géométrique quand [les trois termes] sont tels que le premier est relativement au deuxième comme le deuxième relativement au troisième » $(\dot{\alpha}$

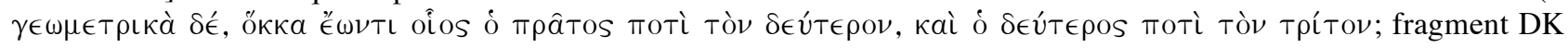
47 B2 ([Die Fragmente der Vorsokratiker ..., 1985], t. I, p. 436, 1. 6-7).

91 V. Merker, p. 00, nn. 00-00,.

92 V. [Alex. in Meteor.], p. 168, 1. 22-23. Mais c'est la seule occurrence dans la paraphrase pourtant très complète qu'il fait de toute la portion centrale de l'argument géométrique. Pour le reste, outre plusieurs expressions avec " $\lambda$ ó $\gamma 0 S^{\prime}$, Alexandre fait usage de la formule euclidienne complète (ii), 10 fois, ou abrégée (iii), 7 fois.

93 V. supra, Merker, pp. 00. 
3. La question du début (a) implique que l'on s'interroge sur le statut des différentes assertions de la section 376 a1-10 :

\begin{tabular}{|c|c|c|}
\hline (12) & 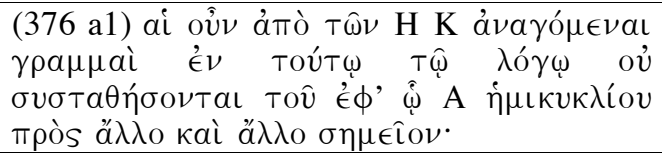 & $\begin{array}{l}\text { Les lignes menées à partir des points } \mathrm{H} \text { et } \mathrm{K} \text { ne se } \\
\text { combineront alors pas, dans ce rapport, en joignant } \\
\text { des points différents du demi-cercle } \mathrm{A} \text {. }\end{array}$ \\
\hline (13) & 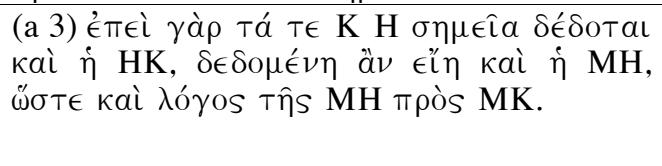 & $\begin{array}{l}\text { Puisqu'en effet les points } \mathrm{H}, \mathrm{K} \text { sont donnés, la droite } \\
\mathrm{HK} \text { le sera aussi, la droite MH doit aussi être } \\
\text { donnée, en sorte que le rapport de MH à MK l'est } \\
\text { aussi. }\end{array}$ \\
\hline (14) & 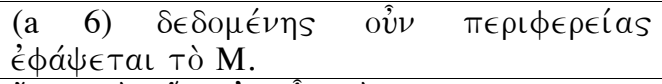 & Le point $\mathrm{M}$ touche donc une circonférence donnée. \\
\hline (15) & 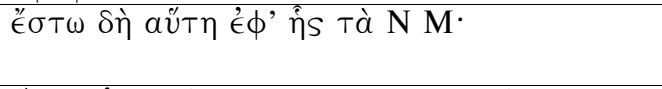 & $\begin{array}{l}\text { Que celle-ci soit celle sur laquelle sont les points } \\
\text { MN; }\end{array}$ \\
\hline (16) & 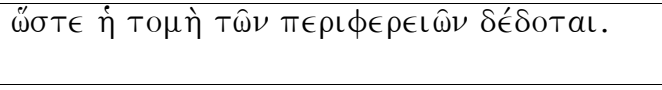 & $\begin{array}{l}\text { par conséquent la section des circonférences est } \\
\text { donnée. }\end{array}$ \\
\hline (17) & 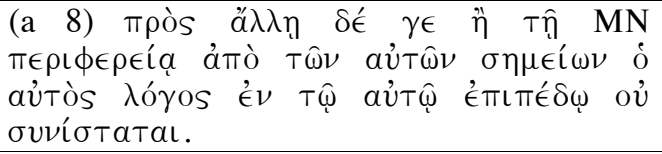 & $\begin{array}{l}\text { Or dans un même plan et à partir des mêmes points } \\
\text { le même rapport ne se constitue pas contre une autre } \\
\text { circonférence que MN. }\end{array}$ \\
\hline
\end{tabular}

Plus précisément, quels sont les liens de l'assertion (17) avec ce qui précède et ce qui suit ? Est-ce l'énoncé de l'assertion prouvée à la suite $(376 \mathrm{a} 10-\mathrm{b} 7=\mathbf{1 8 - 3 9})$ ? Ou bien est-ce la conclusion de la "démonstration" précédente (13-16) ou encore une simple redite de (12), comme le dit un peu vite Alexandre d'Aphrodise?

4. L'explication d'Alexandre est plutôt étrange, mais il faut dire que le texte dont il fait état ${ }^{94}$ est différent de celui que retiennent les éditeurs modernes des Météorologiques; en fait il est bien plus proche d'une variante attestée dans quelques manuscrits ${ }^{95}$ :

\begin{tabular}{|c|c|c|}
\hline (17') & 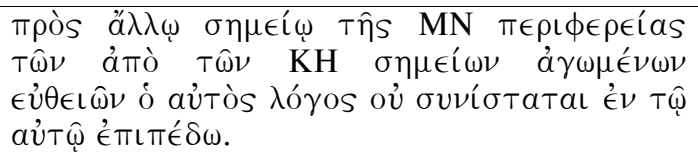 & $\begin{array}{l}\text { Or le même rapport des droites menées à partir } \\
\text { des points } \mathrm{H} \text {, K ne se constitue pas, dans un } \\
\text { même plan, en un autre point de la circonférence } \\
\text { MN. }\end{array}$ \\
\hline
\end{tabular}

Alexandre se croit donc autorisé à dire qu'il s'agit d'une redite de (12). Il faut cependant noter qu'il n'est plus question d'un (autre) point du demi-cercle A (comme dans 12), mais de la circonférence $\mathrm{MN}$, et on ne voit pas alors pourquoi il faut préciser « dans le même plan ». En outre son commentaire laisse complètement en suspens la nature de ce qui vient à la suite puisqu'il a affirmé qu'Aristote a omis la preuve de (12), parce qu'évidente ${ }^{96}$, donc aussi de (17) s'il ne s'agit que d'une redite. Mais alors que contient la séquence $(376 \mathrm{a} 10-\mathrm{b} 7 \mathbf{= 1 8 - 3 9})$ ? De plus que faut-il penser de la portion (376 a3-7 = 13-16) ? Quelle est sa fonction ? Alexandre la commente longuement pour essayer de justifier le fait que HM est donnée, et donc que le rapport HM : MK est donné, mais en commettant une pétition de principe ${ }^{97}$. Il est difficile de dire si c'est l'incompréhension du passage qui a provoqué l'altération du texte ou si l'explication procède en sens inverse mais la lecture d'Alexandre n'est pas recevable ${ }^{98}$.

5. Pour Tannery l'assertion (17) marque le début de l'interpolation, ce qui va plutôt dans le sens de

\footnotetext{
94 [Alex. in Meteor.], p. 167, 1. 6-8. En revanche Alexandre reproduit (12) à l'identique (ibid., p. 165, 1. 5-7).

95 V. l'apparat critique dans [Aristote, Louis, 1982], p. 21 pour la ligne 376 a8 (mss $\mathrm{F}^{2} \mathrm{HN}$ ). Il peut s'agir d'une modification du texte déterminée par la leçon que donne Alexandre.

96 Il produit lui-même cette preuve, p. 165, 1. 21-p. 166, 1. 5 : il choisit un point $\mathrm{N}$ sur le demi-cercle A, autre que M et montre que le rapport HN : NK ne peut pas être le même que le rapport HM : MK car MK et NK sont égales comme rayons, tandis que HN est plus grande ou plus petite que HM selon qu'elle est plus proche ou plus éloignée qu'elle du diamètre (d'après Eucl., Él. III. 7).

97 [Alex. in Meteor.], p. 166, 1. 10-p. 167, 1. 6.

${ }^{98}$ La leçon retenue par les éditeurs modernes (17) - malgré les nombreuses variantes dont témoignent les manuscrits - est quand même plus satisfaisante que celle d'Alexandre (17').
} 
la première hypothèse. Mais alors pourquoi affirmer que l'objectif de l'interpolateur est de montrer qu'il est possible de construire une circonférence solution du problème de lieu, ce qui correspond à la première partie de la preuve d'Apollonius ${ }^{99}$ ? La formulation de (17) fait davantage penser à une assertion d'unicité, en l'occurrence celle de la circonférence $\mathrm{MN}$; c'est pourquoi $\mathrm{M}^{\mathrm{me}}$ Merker rapproche (17) de la seconde partie de la preuve d'Apollonius et non de la première. La lecture qui paraît la plus naturelle est de considérer que les assertions (12) et (17) ne se rapportent pas exactement au même problème :

99 [Tannery, MSc, 1929], p. 57; v. aussi supra la citation de la note 10. 
-(12) affirme l'unicité d'un point tel que M sur la circonférence d'un des demi-cercles de l'horizon; ce qui est justifié dans la séquence (13-16);

-(17) affirme l'unicité de la circonférence MN dans le plan A, pour garantir le fait que le point $\mathrm{M}$ est unique dans le demi-cercle (Cf. 12), comme intersection de deux circonférences complètement déterminées (16). Quelles sont ces deux circonférences ? Pour Alexandre ${ }^{100}$, il s'agit de l'intersection du cercle de centre $\mathrm{K}$ et de rayon KM donné et du cercle de centre $\mathrm{H}$ et de rayon $\mathrm{HM}$ donné ! Mais ceci est incompatible avec l'insertion (15) qui implique que la circonférence MN soit l'une des deux lignes en question. Pour Tricot ${ }^{101}$, il s'agit de l'intersection de l'horizon et de l'arc-enciel MN. Cette interprétation suppose que l'affirmation (14) désignait elle aussi l'arc-en-ciel comme circonférence donnée, sans justification préalable, en particulier sans lien avec (12) dont on ne comprend plus alors la signification ${ }^{102}$. Il n'y a donc pas de doutes : il s'agit de l'horizon et du cercle dit d'Apollonius. Que M soit déterminé c'est d'ailleurs ce que présuppose la spécification "donnée" dans (14). L'assertion (17) introduit donc une justification de la justification ou, comme le dit Tannery, une affirmation complémentaire qui va être prouvée dans ce qui suit. Cette liaison démonstrative exige donc que nous rejetions également (17) comme inauthentique.

6. Dans cette interprétation et contrairement à ce que dit Alexandre, la séquence (14-16) est une justification de (12) et c'est dans (14) que le texte se réfère au théorème d'Apollonius d'une manière plutôt globale, ce à cause de la notation "donnée". Si nous avions : « Le point M touche donc une

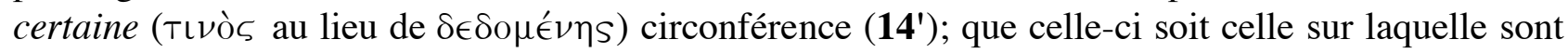
les points $\mathrm{MN}$; de plus, dans ce même plan et à partir des mêmes points, le même rapport ne se constituera pas contre une autre circonférence que MN. Donc ... » nous pourrions dire que l'auteur suit de près la formulation apollinienne : affirmation d'existence (14'); affirmation d'unicité (17). Mais en affirmant, dès (14), que le lieu de M est donné, il a combiné les deux aspects et c'est ce qui a provoqué l'insertion ultérieure de (17) avec la longue interpolation centrale.

7. Pour pouvoir se référer au théorème des lieux plans il faut évidemment s'assurer que l'hypothèse dudit théorème - le fait que le rapport des droites HM : MK est donné - est satisfaite, ce à quoi s'emploient la mystérieuse clause « dans ce rapport » de (12) et la séquence (13) toute entière. Cette dernière est rejetée par Tannery à cause de l'usage du seul lettrage simple. Mais nous avons vu que cet argument est certainement insuffisant. On peut observer qu'elle est formulée dans le langage des Données d'Euclide ${ }^{103}$, ce qui n'a rien non plus d'étonnant si l'on envisage, comme Tannery, qu'il s'agisse d'une insertion postérieure puisque le terme "donnée" apparaît aussi dans (14) et (16). Cela dit, la rédaction de (13) en est très maladroite et son contenu fort discutable :

- Elle passe allègrement du couple (HM, HK) au couple (HM, MK) sans justification (ce qui serait d'ailleurs plutôt délicat).

\footnotetext{
100 [Alex. in Meteor.], p. 167, 1. 1-2.

101 V. [Aristote, Tricot, 1976], p. 210, n. 1.

102 Quant à [Aristote, Louis, 1982], p. 21, n. 2, il se contente de dire qu'il s'agit de l'intersection de l'horizon avec la circonférence $\mathrm{MN}$ ! Cela n'engage à rien.

103 [Knorr, 1986], p. 108-111, fait grand cas de cette coïncidence et en déduit que l'approche des Données euclidiennes n'est pas une invention de l'auteur des Éléments puisqu'il avait été précédé par l'hypothétique auteur aristotélicien qu'il postule. Rappelons que notre collègue n'émet aucune réserve quant à l'intégrité du texte des Météorologiques.
} 
- Par rapport à l'assertion (12) il s'agit de ce que j'appelle une explication postposée ${ }^{104}$, essayant de justifier l'affirmation concernant le rapport HM : MK. Ces explications postposées, dans les textes mathématiques grecs synthétiques, proviennent généralement de remarques marginales insérées dans les marges des manuscrits par des lecteurs cherchant à justifier une affirmation ou une inférence dont ils n'aperçoivent pas la justification ${ }^{105}$. Ici il pourrait s'agir d'une glose marginale

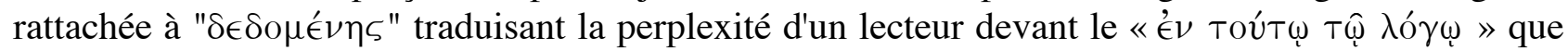
rien ne justifie vraiment, ni avant, ni après et que l'on pourrait traduire : « puisqu'en effet les points $\mathrm{K}, \mathrm{H}$ sont donnés, et [donc] HK, il faudrait que MH soit donnée afin que le rapport de MH à MK le soit ».

- En fait il n'y aucun moyen de justifier le fait que le rapport HM : MK est donné sauf à le postuler ou à poser un principe encore plus puissant, par exemple que $\mathrm{M}$ est donné, ou que l'angle sous HKM est donné106 ...

Il était évidemment plus expédient de dire que les points $\mathrm{H}, \mathrm{K}, \mathrm{M}$ sont (au moins momentanément) donnés. Heath, réagissant à une suggestion de Poske, affirme qu'Aristote ne suppose pas le point $\mathrm{M}$ donné, mais qu'au contraire, il le construit ${ }^{107}$. On aimerait qu'il nous explique comment. Car si c'est comme intersection de deux circonférences, comment construit-on l'arc MN du cercle d'Apollonius sinon en connaissant le rapport HM : MK, ou si l'on préfère D : B ? - Le point de départ de (13) est que $\mathrm{H}$ et $\mathrm{K}$ seulement sont donnés, comme dans le résultat d'Apollonius; ce n'est pas la situation météorologique qui commande cette portion, puisque alors $\mathrm{H}$, $\mathrm{K}, \mathrm{M}$ seraient donnés, mais les hypothèses du théorème de géométrie contenu dans (12).

- Qu'il ne s'agit pas non plus de météorologie dans (12) mais de géométrie, c'est ce que montre

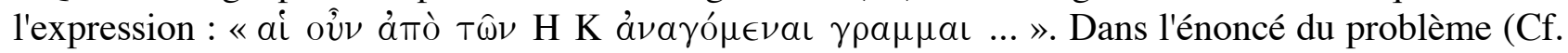

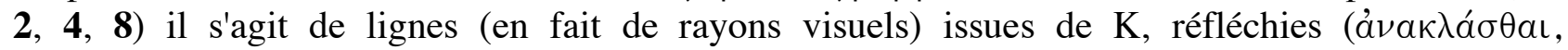

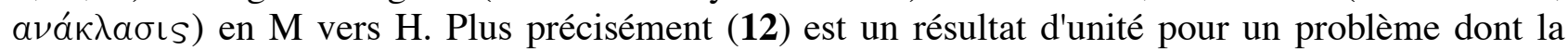
solution - par analyse et synthèse - nous a été conservée par Pappus dans un Lemme pour les Porismes d'Euclide108 : «Un segment de cercle sur la droite AB étant donné, fléchir la ligne ACB dans un rapport donné ${ }^{109}$. La nature seconde de (12) par rapport au problème cité par Pappus rend

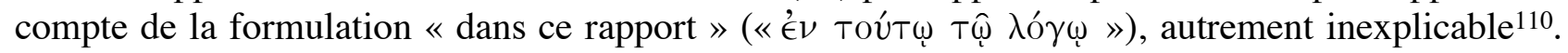
Le principe de la solution du Lemme VII. 29 (la similitude de certains triangles ${ }^{111}$ ) est le même que celui que l'on trouve ici même dans l'interpolation centrale (Cf. 376 a 29-30 = 32) et dans la preuve d'Apollonius $^{112}$. Ce qui, indépendamment de la décision que l'on prend pour le texte des

104 J'appelle « explication postposée » les explications qui inversent l'ordre logique déductif « si p, alors $\mathbf{q}$ » pour dire « $\mathbf{q}$, parce que $\mathbf{p} »$. La forme la plus habituelle en mathématiques, dans l'exposé synthétique (procédant des hypothèses vers les conclusions) est la forme « si $\mathbf{p}$, alors $\mathbf{q}$ ». Dans un exposé synthétique, comme celui d'Euclide ou d'Apollonius, une formule postposée trahit un défaut d'analyse. J'ai introduit cette terminologie dans ma typologie des enrichissements locaux subis par le texte des Éléments. V. [Euclide, 2001], pp. 45-60.

105 Chez Aristote, les explications postposées sont fréquentes car elles sont très pédagogiques et restituent le cheminement du raisonnement là où l'exposé synthétique procède d'une manière souvent élégante mais artificielle. On peut aussi penser que l'auteur de notre passage reproduit une analyse et non une synthèse.

106 Ce que confirment les divagations d'Alexandre à ce sujet. V. [Alex. in Meteor.], p. 166, 1. 10-p. 167, 1. 6 et supra, $\S$ 4.

107 V. [Heath, 1949], p. 188.

108 [Papp., Coll. math.], Livre VII, Prop. 155, § XXIX, p. 904, 1. 17-p. 906, 1. 12, = 28 $8^{\mathrm{e}}$ Lemme pour le second Porisme du Premier Livre des Porismes d'Euclide. V. Annexe I. Le rapprochement entre ce Lemme et le texte des Météorologiques a déjà été fait dans [Knorr, 1986], pp. 140-141, n. 16. Il fait également le lien avec l'utilisation, plutôt

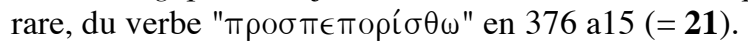

109 L'assertion (12) est relative au cas particulier du demi-cercle; tandis que le problème et la solution cités par Pappus valent pour n'importe quel segment de cercle.

110 Comme l'a bien montré $\mathrm{M}^{\mathrm{me}}$ Merker (pp. 00), voir dans cette constance du rapport une loi de la physique aristotélicienne est une illusion de l'épistémologie moderne déçue de ne pas trouver la loi de la réflexion dans le traitement de l'arc-en-ciel.

111 Mais l'équiangularité des deux triangles est elle-même une conséquence d'une propriété de la tangente à un cercle, celle établie dans Eucl. Él. III. 32; v. [Papp., Coll. math.], p. 906, 1.9 et Annexe I.

112 [Eut. in Apoll.], p. 182, 1. 19-21. V. infra, III, § 8. 
Météorologiques, suggère fortement que ledit principe était connu et mis en œuvre antérieurement à la rédaction des Lieux plans, en particulier par Euclide.

8. Dès lors, pourquoi maintenir (partiellement) la portion (12-16) comme le fait Tannery ${ }^{113}$ ? Celuici s'y rallie sans doute à cause de la présence de deux notations complexes, présence qui constitue

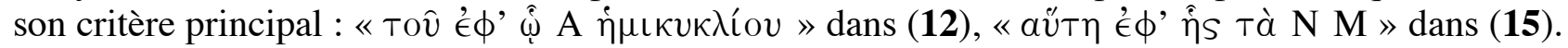
Mais observons que la seconde n'est peut-être pas une vraie notation complexe; en effet on devrait

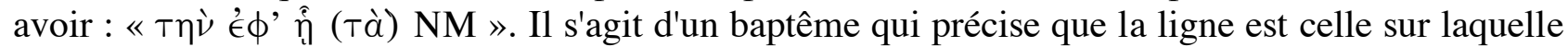
(sens usuel de "€́Tí" + génitif) il y a les points $\mathrm{N}$ et $\mathrm{M}$ (Cf. l'article neutre "Tà"). Reste donc seulement la première notation, pour le demi-cercle $\mathrm{A}$. Bien que cela me conduise à anticiper sur ce qui suit, je dirai que dans la reconstruction que je propose l'interpolation est constituée de la séquence (12)-(39) + (41) et donc le texte authentique présentait la succession suivante : «(10)

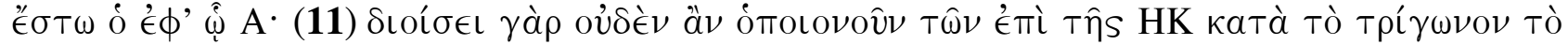

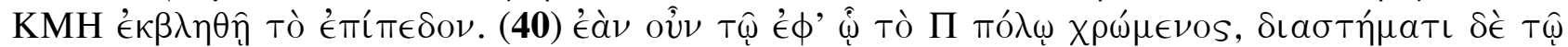

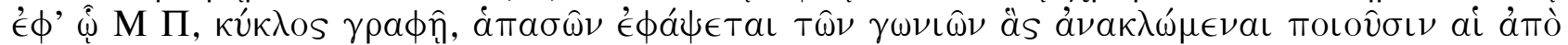

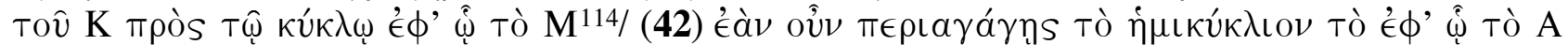

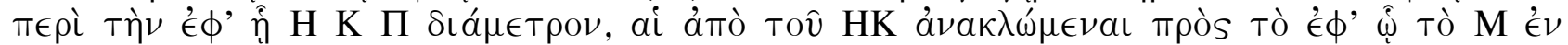

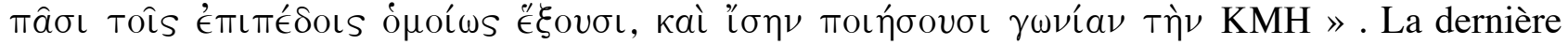
séquence introduisait donc la notation (complexe) pour le demi-cercle A et toute la séquence interpolée a été introduite pour justifier que sa rotation engendre bien un cercle qui n'est autre que celui dont l'arc-en-ciel est une portion. Il n'est donc pas étonnant que la première phrase de ladite interpolation (12 dans cette hypothèse) reprenne elle aussi ladite notation ${ }^{115}$. Par ailleurs j'ai essayé de montrer que certains mathématiciens hellénistiques, peut-être Euclide lui-même, certainement Archimède, utilisaient cette notation complexe; l'argument n'a donc pas la valeur probante que pouvait lui reconnaître Tannery. Sans instruire de procès d'intention, on peut aussi penser que l'illustre historien de la géométrie grecque était enclin à accepter cette portion du texte parce qu'à cause d'elle on pouvait invoquer une loi aristotélicienne de constance du rapport qui justifiait le recours au théorème des Lieux plans, et aussi parce qu'elle permettait ainsi de documenter un point d'histoire de la géométrie préeuclidienne ${ }^{116}$, l'un des soucis majeurs des reconstructions de Tannery. Comme lui je pense que la portion (17-39) fait partie de l'interpolation ou des interpolations ajoutée(s) au texte d'Aristote. Mais, compte tenu de l'analyse qui précède, il me paraît fort probable qu'il faille également y inclure la section (12-17). Reste à voir ce qu'il en est de la fin.

9. L'interpolation centrale se poursuit au moins jusqu'à l'établissement de la proportion contenue dans (39) : ПН : МП :: МП : ПК, soit, pour le dire autrement : si le rapport $\Delta:$ B est donné et si le point $\mathrm{M}$ vérifie $\mathrm{HM}: \mathrm{MK}:: \Delta: \mathrm{B}$ et si le point $\Pi$ est construit selon les indications données dans (21-22), alors МП est la moyenne géométrique de deux droites données $(\Pi \mathrm{H}, \Pi \mathrm{K})$; elle est donc donnée de grandeur et $\mathrm{M}$ appartient donc à un cercle de centre $\Pi$, ce qu'annonçait (24). Déterminer la fin de la partie interpolée revient donc à trancher le problème de l'authenticité des assertions (4041):

\begin{tabular}{|c|c|c|}
\hline (40) & 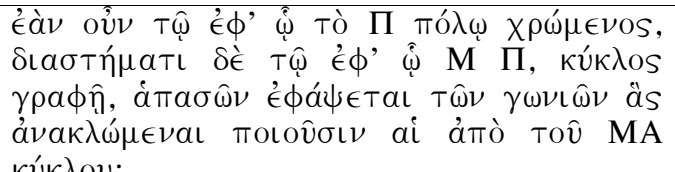 & $\begin{array}{l}\text { Si donc en se servant de } \Pi \text { comme pôle et de } \mathrm{M \Pi} \\
\text { comme intervalle on trace un cercle, il touchera tous } \\
\text { les angles que forment les droites en se réfléchissant } \\
\text { à partir du cercle MA. }\end{array}$ \\
\hline
\end{tabular}

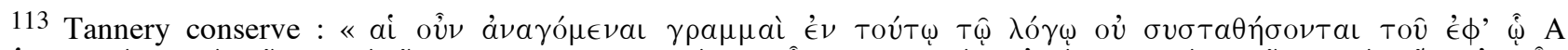

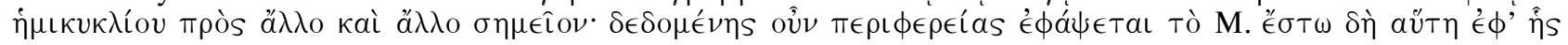

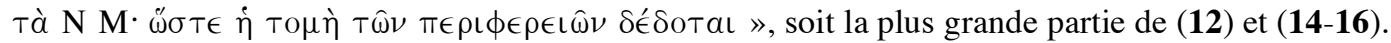

114 V. infra, § 10 et n. 119.

115 Que l'on exclut (11) ou qu'on le maintienne totalement ou partiellement (comme Tannery), cela ne change rien au point que je discute ici.

${ }^{116}$ Sur ce point, v. supra, I, §§ 3-4.
} 

impossible.

10. Pour (40) il y a deux lignes d'interprétation possibles :

- On admet que $\Pi$ est le point qui vient d'être mis en évidence, autrement dit le centre du cercle d'Apollonius (Cf. figure 2), il faudra, comme dans (24), corriger "тó $\lambda \omega$ " en "ќ́ $\nu \tau \rho \omega$ " et considérer (40) comme la conséquence logique de ce qui précède : le tracé dudit cercle dans le plan de l'horizon. Pour défendre cette lecture on fera remarquer, à la suite de Heath ${ }^{117}$, que la prise en compte de la troisième dimension n'intervient qu'avec la révolution du demi-cercle $\mathrm{A}$ et du triangle HMK dans (42). Clairement, si l'on adopte cette interprétation, l'assertion (40) - et (41) qui l'accompagne - est rejetée comme conclusion de l'interpolation laquelle inclut alors l'ensemble (12-41).

- Malgré sa plausibilité, je ne suis pas tout à fait convaincu par cette lecture dans laquelle je perçois deux difficultés :

- si l'on exclut (12-41) le point $\Pi$ sera seulement introduit à l'étape (42) au moment de la

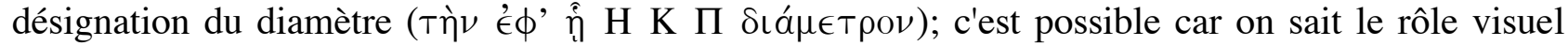
important que joue le diagramme lui-même dans l'introduction des lettrages, mais c'est un peu gênant car, comme le montre la suite de l'argument, la fonction polaire de $\Pi$ est importante; or celui-ci sera seulement introduit, incidemment, comme extrémité du diamètre.

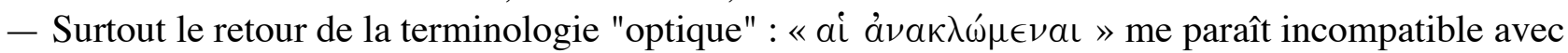
l'interprétation qui ferait de (40) la conclusion de la section purement géométrique (12-39).

Mais y a-t-il vraiment une difficulté à penser que (40) fait référence au tracé de l'arc-en-ciel et donc de considérer que $\Pi$ est bien un pôle comme sur la figure 1 ? Le double emploi avec (42) n'est pas si net : (40) peut représenter l'étape du tracé effectif du cercle selon une procédure canonique de la sphérique et (42) le début de l'argument qui va justifier que l'on a, comme dans le

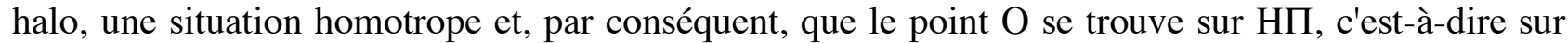
l'horizon. Ce qui démontre que l'arc-en-ciel est alors un demi-cercle. Cette interprétation me paraît possible, que l'on accepte l'authenticité de la portion (43-47) comme $\mathrm{M}^{\mathrm{mr}}$ Merker ou qu'on lui préfère un argument plus expédient ${ }^{118}$. De même, dans l'une ou l'autre des deux interprétations, la

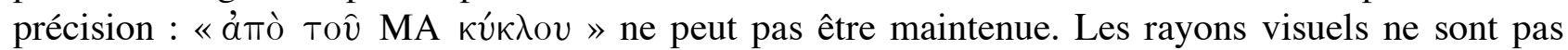
réfléchis à partir du cercle - que l'on n'a d'ailleurs jamais appelé MA - mais à partir du centre K, contre ou sur le cercle, en M, vers l'astre H. De plus s'il s'agit du cercle d'Apollonius un seul rayon visuel s'y réfléchit dans le demi-cercle comme cela a été dit en (12) et est apparemment rappelé en (41). Et si, comme je le crois, il s'agit de l'arc-en-ciel, celui n'est pas contenu dans le plan contenant le cercle MA (l'horizon ?) mais lui est perpendiculaire. Pour ma part je proposerai donc une

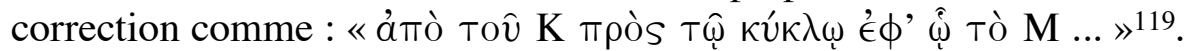

11. Reste l'assertion (41) qui paraît plutôt inutile et peut-être même altérée. Dans la formulation transmise, il s'agit en effet d'un renvoi à l'impossibilité d'une construction : « ce qui était impossible », mais laquelle ? Celle énoncée dans (12), comme le suggère le mot "demi-cercle", ou celle de (17) ? Cette seconde hypothèse est difficile à concilier avec le texte transmis, sauf pour Alexandre qui, rappelons-le, identifie au demeurant les contenus de (12) et (17), en s'appuyant sur une formulation plutôt divergente, formulation que l'on retrouve encore ici même ${ }^{120}$ :

\footnotetext{
117 V. [Heath, 1949], p. 185.

118 V. supra, Merker, pp. 00.

119 Tricot ([Aristote, 1976], p. $212+$ n. 5) corrige : « ... tous les angles que font, par leur réflexion, les lignes partant de $\mathrm{H}$ et $\mathrm{K}$... » en s'inspirant d'[Alex. in Meteor.], p. 170, 1. 1-2.

120 Mais à la différence de ce que l'on observe pour (17), cette altération n'a pas été introduite dans le texte même des Météorologiques. Cf. supra, n. 95.
} 
Car s'il ne les touche pas il se produira que les droites fléchies à partir des mêmes points jusqu'en un autre point dans le même plan, auront, l'une relativement à l'autre, le même rapport, ce qui est impossible.

Si l'on admet à la fois que (40) porte sur le tracé de l'arc-en-ciel et que (41) est un renvoi à (12) ${ }^{121}$, il interrompt inutilement la démonstration qui, après le tracé du cercle de l'arc-en-ciel, argumente maintenant sur la position du centre pour savoir s'il s'agit ou non d'un demi-cercle. On n'en est plus aux questions de la nature circulaire du lieu. Dans ce cas également on doit conclure que l'assertion est inauthentique.

12. Finalement, pour résumer ce qui précède, je proposerai l'hypothétique scénario suivant :

- (i) Le texte d'Aristote correspondait, peut-être avec des variantes locales plus ou moins importantes, avec ce que nous transmettent les séquences (1-6), (7-11), (40), (42), (43-47), soit une petite vingtaine de lignes dans l'édition de Bekker, comparable en taille aux deux autres parties de la démonstration (16 et 17 lignes respectivement).

- (ii) Une annotation marginale a été introduite, motivée par un présupposé des constructions contenues dans les assertions (40), (42), lesquelles admettent l'unicité du point M dans le demicercle générateur. Cette scholie justifiait ladite unicité à laquelle font écho, chacune à leur manière

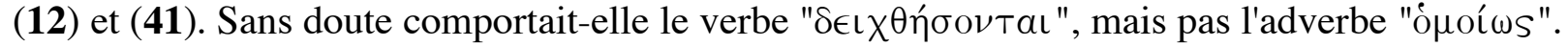

- (iii) En même temps, ou dans une étape ultérieure, on a voulu justifier l'affirmation d'unicité de la scholie, ce qui aurait engendré la section (12-16). Celle-ci se serait ensuite trouvée intégrée dans le texte, comme cela se produit souvent avec les annotations marginales quand elles sont confondues avec des portions de textes oubliées et à rétablir. Il n'est pas certain que cette insertion ait provoqué la disparition de la scholie attachée à (40), (42). Chacune de ses altérations ne s'est pas non plus nécessairement produite dans toutes les copies.

- (iv) Une fois la question posée du rapport HM : MK il apparaissait que l'affirmation (14) en terme de « circonférence donnée » faisait à son tour référence à une unicité, celle du lieu MN. Ce qu'un second ajout - à l'origine de la preuve accessoire (17-39) - s'est efforcé de justifier, en utilisant maladroitement la lettre $\Pi$ pour désigner le centre du cercle dit d'Apollonius.

- (v) Ce second ajout s'est retrouvé lui aussi inséré dans le texte aboutissant à l'usage de la même lettre pour deux points différents; d'où la correction "pôle", tout aussi maladroite, introduite ensuite dans (24). La première scholie a également été intégrée dans le texte (41) et dès lors perçue comme

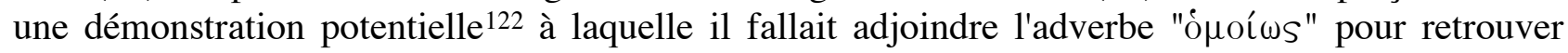
l'une de ses formules habituelles.

Ce scénario est purement logique. Il est impossible de savoir dans quelle mesure il correspond à ce qui s'est vraiment passé au cours de l'histoire de notre texte. Celui-ci résulte peutêtre de la confrontation et de la compilation de versions légèrement différentes au moment de la constitution des manuscrits translittérés, au IX ${ }^{\mathrm{e}}$ siècle à Constantinople, comme cela semble s'être produit pour le texte des Éléments d'Euclide ${ }^{123}$. Quoi qu'il en soit, il m'incline à penser que la thèse selon laquelle la portion $(\mathbf{1 2 - 3 9})+(\mathbf{4 1})$ est apocryphe est plutôt plausible. On peut même douter de l'authenticité de (40) ou, à l'inverse, vouloir conserver la section (12-16). Si l'on ne veut pas trancher, on dira qu'il y a deux ${ }^{124}$ voire quatre reconstructions possibles, mais celle que j'ai indiquée a mes préférences. Bien entendu elle a comme conséquence que le texte original des Météorologiques ne faisait aucune allusion au théorème des Lieux plans d'Apollonius.

121 Comme l'affirment [Tannery, MSc, 1929], p. 58), [Aristote, Tricot, 1976], p. 213, n. 1 et [Aristote, Louis, 1982], p. 23, n. 2.

122 Sur les différentes modalités stylistiques utilisées par les géomètres grecs pour abréger les démonstrations, voir [Euclide, 2001], en particulier, pp. 45-47.

123 V. par exemple [Rommevaux, S., Djebbar, A., Vitrac, B., 2001], en particulier pp. 254-255 et p. 278. 


\section{La comparaison avec Apollonius et la nature de l'interpolation centrale}

1. La reconstruction que je viens de proposer est évidemment en désaccord avec les analyses de Heath, Picolet et Knorr lesquelles, malgré les nuances qui les distinguent, ont en commun de ne pas remettre en cause l'intégrité du chapitre III. 5 des Météorologiques. Elle diverge également de celle proposée par Paul Tannery quant à l'extension des portions retenues comme authentiques - en particulier Tannery conservait $(\mathbf{1 2}, \mathbf{1 4}, \mathbf{1 5}, \mathbf{1 6}, \mathbf{4 1})$ - mais surtout quant à la nature de ce qui se trouve dans les portions rejetées. C'est le point sur lequel je voudrais revenir maintenant, autrement dit essayer de répondre à la troisième et dernière question posée plus haut.

2. Contrairement à ce qu'affirme Tannery, la portion qu'il rejette (17-39) n'est pas à peu près identique à la démonstration, ou même à la seule première partie de la preuve, d'Apollonius. Au demeurant, telle qu'elle est transmise, ladite portion paraît assez peu cohérente, surtout s'il faut y voir la reproduction (partielle) d'une démonstration géométrique. L'explication que l'on pourrait en proposer après Tannery - c'est une interpolation - ne vaut pas à elle seule : ce n'est pas parce qu'un texte est non authentique (i.e. non aristotélicien) qu'il est incohérent - surtout s'il s'agit d'un texte emprunté à un ouvrage de géométrie. Ce n'est pas non plus parce qu'il est censé être emprunté à un texte plutôt ancien (Hermotime ?). La rigueur des preuves que nous connaissons par les fragments et témoignages se rapportant aux travaux des principaux mathématiciens préeuclidiens, par exemple Hippocrate de Chio ou Archytas de Tarente, est tout à fait comparable à celle des démonstrations des Éléments; elles n'ont pas grand-chose à voir avec l'imbroglio que nous transmet le texte manuscrit des Météorologiques.

3. Comme le suggère le scénario exposé ci-dessus je crois que l'état du texte est le résultat de la concaténation de plusieurs annotations marginales, - certaines plus importantes que d'autres - , ne datant certainement pas toutes de la même période, et qui ont été progressivement intégrées dans le texte. Parmi ces gloses, l'une d'elles, même s'il ne s'agit pas vraiment de la première partie du théorème d'Apollonius, comme le disent Tannery et Heath, mérite notre attention. Elle représente sans doute le travail d'un commentateur des Météorologiques qui a l'ambition de compléter les affirmations aristotéliciennes à partir de certains résultats géométriques connus de son temps. Pour le dire autrement on peut essayer d'isoler un noyau mathématiquement cohérent dont la comparaison avec la preuve rapportée par Eutocius permet même d'améliorer, et sans doute de restaurer, cette dernière.

4. Le noyau en question est constitué des séquences 376 a8-b1 $(=\mathbf{1 7 - 3 5})$ et 376 b3-7 (= 38-39).

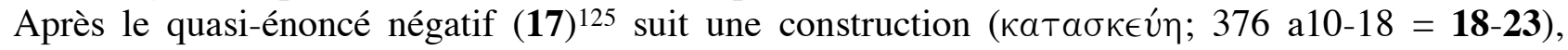
laquelle est à peu près la même que celle d'Apollonius-Eutocius (Annexe II, D). Nous partirons du texte transmis par Eutocius, un peu plus explicite.

Le rapport donné est celui de deux droites inégales $\Gamma: \Delta$, et une médiété géométrique est construite $\Delta: \Gamma:: \Gamma: \Xi$, autrement dit on introduit la troisième proportionnelle $\Xi$ à $(\Delta, \Gamma)(\mathrm{Cf}$. Eucl. $E ́ l$. VI, 11). Dans les Éléments, on dit que le rapport $\Xi: \Delta$ est le rapport doublé de celui de $\Gamma$ à $\Delta$ (en termes modernes c'est le carré) et il est établi que c'est aussi le rapport du carré décrit sur $\Gamma$ au carré décrit sur $\Delta$ (Cf. Eucl. Él. VI, 20 Porisme). En fait le lecteur vérifiera facilement qu'ici $\Xi: \Delta$ est le rapport des droites AZ : ZB (resp. НП : ПK chez Aristote). Il est donc expédient de le séparer (Cf. Eucl. Él. V, Df. 15) pour produire le rapport AZ - ZB : ZB, autrement dit AB : BZ. En effet, si celui-ci est connu, puisque $\mathrm{AB}$ est donnée, on connaîtra $\mathrm{BZ}$ (resp. KП chez Aristote), donc le point $\mathrm{Z}$ (resp. $\Pi$ chez Aristote), centre du lieu cherché. D'où l'idée d'écrire $\Xi$ sous la forme $\Delta+\mathrm{E}$ (resp. $\mathrm{B}$ $+\mathrm{Z}$ chez Aristote), car le rapport séparé de $\Delta+\mathrm{E}: \Delta$ est simplement $\mathrm{E}: \Delta$ (resp. $\mathrm{Z}: \mathrm{B}$ chez Aristote).

124 V. Merker, pp. 00.

125 De tels énoncés négatifs existent aussi dans les Éléments ; v. par ex. I. 7; III. 23. En conséquence la preuve est généralement indirecte sans ecthèse ni diorisme mais commençant directement par la construction, comme ici. V. [Euclide, 1990], p. 210, n. 46. 
Ceci justifie la manière de définir BZ (resp. KП dans 22) comme quatrième proportionnelle à $(\mathrm{E}, \mathrm{AB}, \Delta)($ resp. Z, KH, B).

5. Dans cette portion, les différences entre nos deux textes se limitent au lettrage, avec la correspondance suivante :

\begin{tabular}{|c|ccccccc|}
\hline "Aristote" 126 & $\mathbf{H}$ & $\mathbf{K}$ & $\mathbf{M}$ & $\boldsymbol{\Delta}$ & $\mathbf{B}$ & $\mathbf{Z}$ & $\boldsymbol{\Pi}$ \\
\hline Apollonius & $\mathbf{A}$ & $\mathbf{B}$ & $\boldsymbol{\Theta}$ & $\boldsymbol{\Gamma}$ & $\boldsymbol{\Delta}$ & $\mathbf{E}$ & $\mathbf{Z}$ \\
\hline
\end{tabular}

et au fait que le point $\mathrm{M}$ est supposé être sur le lieu cherché, et le rapport $\mathrm{MH}$ : MK supposé donné. C'est donc à partir de lui que $\Delta \mathrm{B}(\Delta+\mathrm{B})$ est découpée (19) ${ }^{127}$, alors que chez Apollonius c'est l'inverse. Puisque $M$ est situé sur le lieu, il suffit d'en trouver le centre $\Pi$ (Cf. 24; je ne reviens pas sur la confusion avec le pôle de la sphère). Apollonius doit construire le centre $\mathrm{Z}$ et le rayon $\mathrm{ZK}=$ $\mathrm{H}$. De ces différences, il nous faut conclure que le propos de l'auteur est d'adapter son propos au texte d'Aristote. La construction qui paraît plutôt abstruse dans les Météorologiques doit ce caractère d'une part à cette volonté d'adaptation, d'autre part au fait qu'elle présuppose une analyse que l'auteur de l'interpolation, pas plus qu'Apollonius-Eutocius d'ailleurs, n'a cru bon de rapporter. Mais le fait qu'une autre construction (au moins) soit possible, celle proposée par Simson à partir d'Euclide, Él. VI. 3128, prouve que nos deux textes procèdent très certainement d'une source mathématique commune.

6. Pour établir que le point $\Pi$ est le centre du cercle-lieu l'auteur "aristotélicien" proclame que les droites $(\mathrm{KH}, \mathrm{K \Pi}, \Pi \mathrm{M})$ sont dans le même rapport que $(\mathrm{Z}, \mathrm{B}, \Delta)(\mathbf{2 5})$, étant entendu que, par construction, on a déjà : $\mathrm{Z}: \mathrm{KH}:: \mathrm{B}: \mathrm{K}$.

D'où l'on déduit immédiatement (Eucl. Él. V. 12) :

$\mathrm{Z}+\mathrm{B}: \mathrm{HK}+\mathrm{K} \Pi:: \mathrm{B}: \mathrm{K}$, soit Z + B : HП :: B : KП et par permutation (Eucl. Él. V. 16) :

$\mathrm{Z}+\mathrm{B}: \mathrm{B}:: \mathrm{H \Pi}: \mathrm{K \Pi}$.

À cause de la médiété géométrique : $\mathrm{B}+\mathrm{Z}: \Delta: \Delta: \mathrm{B}$, on voit que (KH, KП, ПM) sont dans le même rapport que $(\mathrm{Z}, \mathrm{B}, \Delta)$ si et seulement si $\Pi \mathrm{M}$ est moyenne géométrique entre $\Pi \mathrm{K}$ et $\Pi \mathrm{H}(\mathrm{Cf}$. 39). Cette réduction $(\mathbf{2 5} \rightarrow \mathbf{2 4})$ qui reste plus ou moins implicite chez l'auteur aristotélicien ${ }^{129}$ correspond à la manière dont Apollonius introduit le rayon $\mathrm{H}$ du cercle-lieu à construire comme moyenne des droites (AZ, ZB).

7. Les démarches des deux auteurs divergent davantage dans ce qui suit :

- L'auteur aristotélicien propose une démonstration indirecte (26-37) de la proportion (25) en introduisant une "sixième" proportionnelle, ПP, à (Z, B, $\Delta, \mathrm{KH}, \mathrm{K \Pi )}$. Si elle n'est pas égale à ПM, elle sera ou plus grande ou plus petite. Alexandre précise qu'on la choisit plus petite ce qui est compatible avec la formule de démonstration potentielle insérée dans (38). Par définition on a donc :

$\mathrm{HK}: \mathrm{Z}:: \mathrm{K \Pi}: \mathrm{B}:: \Pi \mathrm{P}: \Delta(\mathbf{2 9})$,

et donc ПР est moyenne géométrique entre $\Pi \mathrm{K}$ et $\Pi \mathrm{H}(\mathbf{3 0})$. Il faut montrer que cela conduit à une impossibilité (Cf. 38).

\footnotetext{
126 Remarquons que le lettrage du Chapitre III. 5, dans la partie « exposé du problème météorologique », est de type

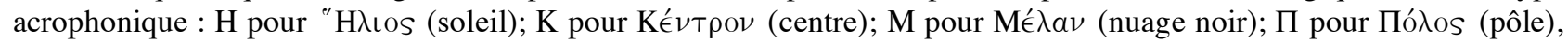
ce qui confirme la position originelle assignée à cette lettre. Avec l'introduction des droites B, $\Delta$ (18) ceci n'est pas possible. L'usage des géomètres consiste, dans la plupart des cas, à suivre l'ordre alphabétique. L'auteur aurait donc pu prendre les droites B, $\Gamma$; mais on remarquera que la lettre $\Gamma$ est utilisée dans la désignation de l'horizon dans le second cas de figure $(376$ b29 = 48). Ceci est peut-être un indice supplémentaire que la partie centrale a été introduite après coup.

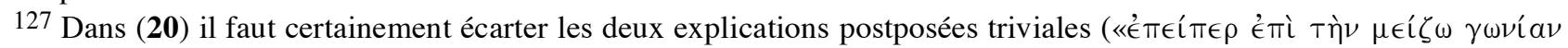

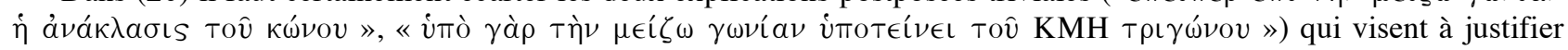
que D est plus grande que B. Outre la caractérisation stylistique (v. supra n. 104), on notera le retour intempestif et pour

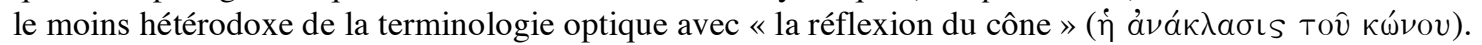

128 Cf. supra n. 21.

129 En fait la réduction apparaît au cours de la preuve indirecte qui suit, dans l'étape (30), sans expliciter les usages de Eucl. V. 12, 16 que nous avons fait apparaître ci-dessus.
} 
- La propriété correspondante dans le texte d'Apollonius-Eutocius : $\Theta Z$ est moyenne géométrique entre $\mathrm{AZ}$ et $\mathrm{ZB}$ est une simple reformulation des conditions de définition du cercle que l'on vient de tracer. En revanche il faut vérifier que tout point $\Theta$ dudit cercle vérifie la condition $A \Theta: \Theta B$ :: $\Gamma$ : $\Delta$, donc que le lieu cherché comprend (au moins) ledit cercle.

8. C'est en ce point de leurs démarches respectives que nos deux auteurs vont faire appel au principe qui sous-tend toute cette affaire, principe que l'on retrouve également dans la configuration du segment de cercle qu'expose Pappus dans son Lemme 29 pour les Porismes d'Euclide (Cf. Annexe I). On peut aussi l'énoncer en termes de triangles correspondant à la généralisation de la célèbre configuration du triangle rectangle et des moyennes géométriques que l'on trouve dans le porisme de la Proposition VI. 8 des Éléments, et qui est la suivante :

Le triangle $\mathrm{A} \Theta \mathrm{Z}$ est rectangle en $\Theta$ et $\Theta B$ est la hauteur relative à $\mathrm{AZ}$.

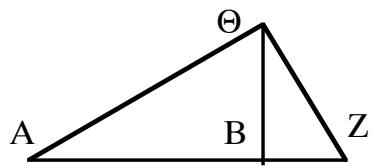

Alors les trois triangles rectangles $\mathrm{A} \Theta \mathrm{Z}, \Theta \mathrm{BZ}, \mathrm{AB} \Theta$ sont semblables et :

- $\Theta B$ est la moyenne géométrique de $\mathrm{AB}, \mathrm{BZ}$

(en termes modernes $\Theta^{2}=A B \cdot B Z$ )

- $\mathrm{Z} \Theta$ est la moyenne géométrique de $\mathrm{ZB}, \mathrm{ZA}\left(\mathrm{Z}^{2}=\mathrm{ZB} \cdot \mathrm{ZA}\right)(*)$.

- $\mathrm{A} \Theta$ est la moyenne géométrique de $\mathrm{BA}, \mathrm{AZ}\left(\mathrm{A} \Theta^{2}=\mathrm{BA}\right.$. $\left.\mathrm{AZ}\right)$.

Par conséquent $\mathrm{A}^{2}: \Theta \mathrm{B}^{2}:: \mathrm{AZ}: \mathrm{BZ}(* *)$.

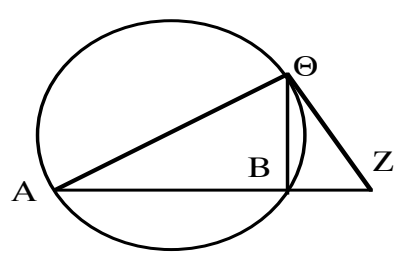

L'égalité $\left.\mathrm{ZO}^{2}=\mathrm{ZB} \cdot \mathrm{BA}\right)(*)$ peut recevoir une interprétation géométrique. Considérons le cercle circonscrit au triangle $\mathrm{AB} \Theta$ : ZB.BA est la puissance du point $\mathrm{Z}$ par rapport à ce cercle et donc, grâce à (*), la droite $\mathrm{Z} \Theta$ est la tangente issue de $\mathrm{Z}$ au dit cercle en $\Theta^{130}$.

Ceci permet une généralisation (partielle) de la situation géométrique ci-dessus aux cas où les triangles ne sont plus rectangles. Soit le triangle $\mathrm{A} \Theta B$ quelconque, non isocèle, ainsi que son cercle circonscrit. On trace la tangente $\Theta Z$ au point $\Theta$ qui coupe le prolongement de $A B$ en $Z$.

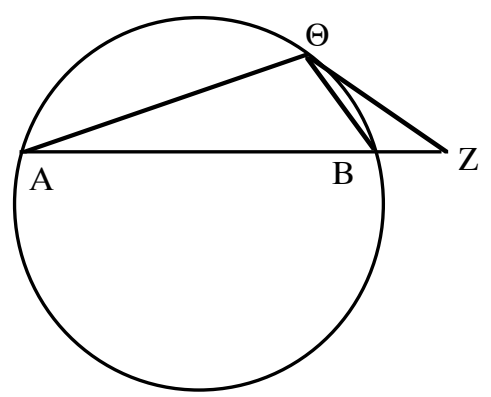

Grâce à Eucl. III. 32 on sait que l'angle sous Z $\Theta B$ est égal à l'angle inscrit dans le segment, celui sous $\Theta A B$.

Puisque les deux triangles $\mathrm{A} \Theta \mathrm{Z}, \Theta B Z$ ont aussi l'angle $\mathrm{Z}$ en commun, ils seront équiangles (Eucl. I. 32), donc semblables.

D'où : $\mathrm{ZA}: \mathrm{Z \Theta}:: \mathrm{Z \Theta}: \mathrm{ZB}:: \mathrm{A} \Theta: \Theta B$.

Et donc certaines des précédentes identités restent valides, notamment :

(*) $\mathrm{Z} \Theta$ est la moyenne géométrique de $\mathrm{ZB}, \mathrm{ZA}$
et $\mathrm{ZA}: \mathrm{ZB}\left(:: \mathrm{ZA}^{2}: \mathrm{Z \Theta}^{2}\right):: \mathrm{A}^{2}: \mathrm{OB}^{2}(* *)^{131}$.

Cela étant, on peut mettre entre parenthèses la situation «segment de cercle / tangente » et raisonner seulement en termes de triangles :

soit deux triangles tels que $\mathrm{A} \Theta Z, \Theta B Z$ ayant l'angle $\mathrm{Z}$ en commun avec $\mathrm{Z}$ sur le prolongement de $\mathrm{AB}$. Si $\mathrm{Z} \Theta$ est moyenne géométrique de $\mathrm{ZA}, \mathrm{ZB}$, comme chez Apollonius-Eutocius, les deux triangles $\mathrm{A} \Theta Z, \Theta B Z$ (resp. HРП, РКП) sont semblables d'après Eucl. VI. 6, 4. D'après ce qui précède, on a donc :

$$
\mathrm{AZ}: \mathrm{Z \Theta}:: \mathrm{A} \Theta: \Theta \mathrm{B} \text { et } \mathrm{AZ}: \mathrm{ZB}:: \mathrm{A} \Theta^{2}: \Theta^{2}(* *) \text {. }
$$

Puisque, par construction, $\mathrm{AZ}: \mathrm{Z} \Theta:: \Gamma: \Delta$, on a bien établi que $\mathrm{A} \Theta: \Theta \mathrm{B}:: \Gamma: \Delta$ et tout point $\Theta$ du cercle appartient bien au lieu cherché. L'auteur aristotélicien fait le même raisonnement (31-33)

130 Pour la formulation ancienne, en termes d'aires, v. Eucl., Él. III. 37.

${ }^{131}$ En revanche le triangle $\mathrm{A} \Theta \mathrm{B}$ n'est plus semblable aux deux autres et les autres identités de VI. 8 ne subsistent pas. Si l'on veut poursuivre dans cette voie, on peut introduire la droite $B \Lambda$ parallèle à $A \Theta$. Alors les triangles $A \Theta B$ et $\Theta B \Lambda$ sont semblables et $\Theta B$ est moyenne proportionnelle de $\mathrm{A} \Theta$ et $\mathrm{B} \Lambda$. C'est la démarche (inutilement compliquée) suivie dans la preuve rapportée par Eutocius. V. infra, $\$ 9$. 
avec les triangles НРП, РКП puisque ПР est la moyenne géométrique de $\mathrm{H \Pi}$ et ПК. On a donc :

НП : ПР :: НР : КР :: $\Delta$ : В (Cf. 35).

9. Fait particulièrement remarquable quoique sans doute contingent, nos deux textes "dérapent" pratiquement à la même étape de leur progression :

- Dans la preuve transmise par Eutocius, après avoir établi la similitude des triangles A $\Theta Z, \Theta B Z$ (Annexe II, E), au lieu d'en déduire immédiatement le résultat cherché comme nous l'avons montré ci-dessus, l'auteur s'engage dans un long détour, complètement inutile, avec construction auxiliaire de la droite $\mathrm{B} \Lambda$ parallèle à $\mathrm{A} \Theta$ (Annexe II, F) pour montrer que les triangles $\mathrm{A} \Theta \mathrm{B}$ et $\mathrm{B} \Theta \Lambda$ sont semblables, puis il compare les différents rapports au rapport $\mathrm{A} \Theta$ : $\mathrm{B} \Lambda$ qu'il élimine ensuite (Annexe II, G) !

Comme l'a fait remarquer Knorr, il est fort peu vraisemblable qu'une telle faiblesse puisse être attribuée à un géomètre du calibre d'Apollonius ${ }^{132}$. Sans doute faut-il penser que le texte original de celui-ci ne donnait guère de détails et que des rééditeurs ou des scholiastes ont tenté de les restituer, non sans maladresse.

- Chez l'auteur aristotélicien, la situation est pire encore puisque le texte devient apparemment incohérent. En effet, ayant établi que les droites jointes (HP, KP) seront dans le rapport de $\Delta$ à $\mathrm{B}$ et après avoir rappelé qu'il en est de même des droites (HM, MK) (34), il poursuit :

\begin{tabular}{|c|c|c|}
\hline (36) & 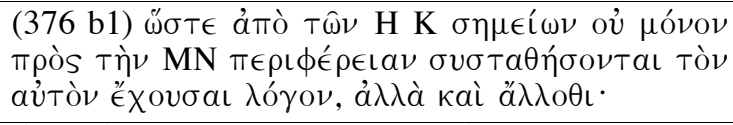 & $\begin{array}{l}\text { De sorte qu'à partir des mêmes points } \mathrm{H} \text { et } \mathrm{K} \text { elles se } \\
\text { combineront non seulement contre la circonférence } \mathrm{MN} \\
\text { avec le même rapport, mais aussi ailleurs; }\end{array}$ \\
\hline (37) & 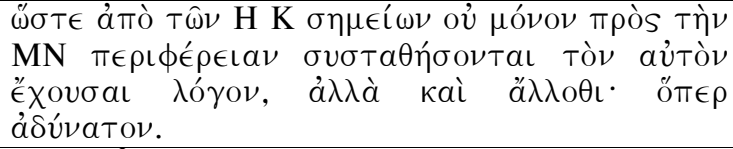 & ce qui est impossible. \\
\hline (38) & 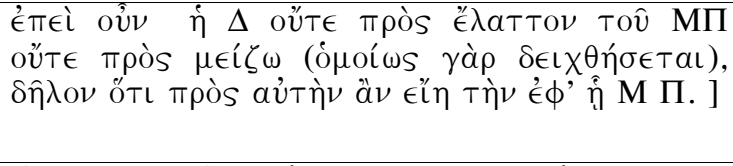 & $\begin{array}{l}\text { Puis donc que } \mathrm{D} \text { n'est tel ni par rapport à une droite plus } \\
\text { petite, ni par rapport à une droite plus grande (car cela } \\
\text { serait démontré semblablement), il est évident que ce } \\
\text { sera à l'égard de MП elle même. }\end{array}$ \\
\hline (39) & 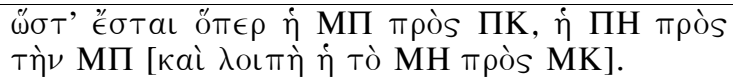 & $\begin{array}{l}\text { De sorte que } \Pi H \text { est à } \mathrm{M \Pi} \text { ce que } \mathrm{M \Pi} \text { est à } \Pi \mathrm{K} \text { [et } \\
\text { enfin } \mathrm{MH} \text { est à } \mathrm{MK} \text { ce que } \mathrm{M \Pi} \text { est à } \Pi \mathrm{K}] \text {. }\end{array}$ \\
\hline
\end{tabular}

L'assertion (38) est clairement la conclusion du raisonnement engagé à partir de (26) pour établir, de manière indirecte, la proportion (25) laquelle est effectivement ré énoncée dans (39). Dans l'état du texte transmis, il faudrait donc que la contradiction obtenue en supposant $\Pi Р<\Pi M$ soit explicitée dans les assertions (36-37), ce que semble confirmer, à première vue, la clause « ce qui est impossible » (37). Or l'assertion (36) ne fait que rappeler ce que l'on cherche à démontrer depuis

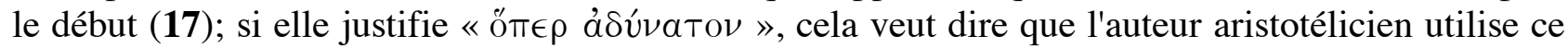
qu'il cherche à démontrer ${ }^{133}$ dans la démonstration ${ }^{134}$ ! En outre l'hypothèse du raisonnement indirect : que ПР est plus petite que ПM, n'est même plus utilisée pour obtenir la contradiction. C'est un peu fort.

132 [Knorr, 1986], p. 104 + n. 12, p. 140. Knorr envisage l'hypothèse que la démonstration d'Apollonius aurait été altérée par Eutocius. À noter que Pappus, dans la notice qu'il consacre aux Lieux plans ([Papp., Coll. math.], L. VII, p. 664, 1. 8-9) fait état d'ajouts apportés par un certain Charmandros, inconnu par ailleurs (voir toutefois l'hypothèse proposée par [Ver Eecke, 1982], p. 497, n. 3). Sans doute s'agit-il d'un rééditeur et/ou commentateur du traité d'Apollonius dont pourrait dépendre Eutocius.

133 D'une manière quelque peu sibylline, [Knorr, 1986] remarque : « But why this should be an apparent contradiction is not quite clear ... Otherwise, he seems to beg the question ... »(pp. 103-104).

134 Pour [Aristote, Tricot, 1976], p. 212, n. 3 les assertions (36-37) appliquent (17), alors qu'elles appartiennent à la preuve de (24) qui sert à établir ... (17). Même chose dans [Aristote, Louis, 1982], p. 23, n. 1). Le cas d'Alexandre ([Alex. in Meteor.], p. 169, 1. 23-25) est encore plus amusant. Son texte, ici comme en deux autres endroits (v. supra, II, $\mathrm{C}, \S 4$ et 11), n'est pas tout à fait le même : il est libellé en terme de «point du demi-cercle » et (36) devient une évocation de (12), dont (17), rappelons-le, n'est qu'une redite pour Alexandre. Pour (37) il dit plus précisément : « ce

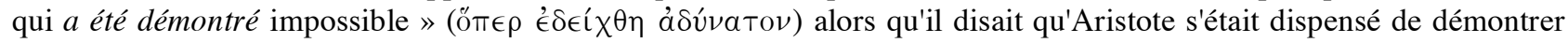

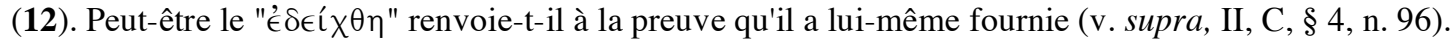


10. Pour essayer de justifier l'état du texte il y a sans doute plusieurs possibilités. J'en vois au moins deux :

- Le lecteur aura certainement remarqué que la démarche de l'auteur aristotélicien est plus proche d'une analyse régressive que d'une synthèse comme celle que transmet la preuve d'ApolloniusEutocius. C'est particulièrement visible dans les transitions $24 \rightarrow \mathbf{2 5}, \mathbf{3 1} \rightarrow \mathbf{3 2}, \mathbf{3 3 - 3 4} \rightarrow \mathbf{3 5}$. Il se pourrait donc que notre interpolation centrale s'inspirât d'une source mathématique qui exposait successivement analyse et synthèse du problème et que la portion conservée de la preuve accessoire soit en fait tronquée. L'assertion (36) aurait pu figurer dans la seconde partie de la preuve, voire en être le diorisme intermédiaire, mais cela suppose que sa formulation ait été altérée, ce qui est au demeurant vraisemblable, pour essayer de redonner un sens à un texte mutilé. Cela dit, cette hypothèse est complètement $a d$ hoc et elle oblige à suppléer davantage de portions de texte ...

- Il me semble donc plus simple de considérer (36) comme une glose marginale due à un lecteur qui ne comprenait pas que la contradiction cherchée était à peu près évidente ou qui ne percevait plus l'intention de la séquence (26-39).

En effet, on a établi que HP : PK :: HM : MK, et comme dans la situation propre à la sphère "météorologique" on a KM = KP, cette proportion est impossible puisque par hypothèse $\Pi \mathrm{P}<\Pi \mathrm{M}$. Si donc on élimine (36), il reste une séquence $(\mathbf{3 5}),(37-39)$ qui constitue une fin de démonstration à peu près correcte. Dans cette interprétation, on retrouve l'idée que l'auteur vise à compléter l'exposé aristotélicien dont il utilise ici une hypothèse forte, à savoir que KM $=\mathrm{KP}$. Sans utiliser cette hypothèse je ne vois pas comment conclure à l'impossibilité. Cela reviendrait à établir en toute généralité (i.e. sans hypothèse supplémentaire) le résultat d'unicité qui constitue la deuxième partie de la preuve d'Apollonius-Eutocius (Annexe II, J-N). On pourrait espérer que celle-ci nous fournisse quelques éclaircissements sur la stratégie que l'auteur aristotélicien aurait pu adopter. Malheureusement cette seconde partie me semble complètement corrompue ${ }^{135}$ et elle n'est d'aucun secours.

\section{Conclusions}

Parmi les différentes descriptions et explications antérieures du chapitre III. 5 des Météorologiques, je crois que celle de Tannery est la moins mauvaise, même si les arguments utilisés ne sont pas toujours probants. Elle doit être aussi assez fortement modifiée, tout particulièrement sur deux points :

- Je crois qu'il faut admettre une construction complexe et progressive de ce texte, intégrant diverses annotations marginales aussi bien dans la partie authentiquement aristotélicienne que, plus tard, dans des ajouts déjà intégrés au texte.

- J'ai essayé de montrer aussi que le noyau de l'interpolation centrale, assez facile à isoler (grâce à sa formulation idiosyncrasique des proportions), n'est pas la reproduction quelque peu servile de la démonstration de l'assertion directe du théorème d'Apollonius (existence du lieu) mais qu'il s'agit du travail d'un commentateur cherchant à compléter les indications aristotéliciennes. Il suppose connu un point du lieu, $\mathrm{M}$, et sans doute sa nature : un cercle. Il en établit l'unicité en montrant celle du centre $\Pi$ (et donc du rayon $\Pi \mathrm{M}$ ). Il combine donc la construction de la première partie du théorème d'Apollonius et le résultat converse d'unicité qu'il peut établir plus simplement grâce à l'hypothèse supplémentaire que fournit la situation traitée par Aristote.

Cette description n'est pas la seule possible et, comme mes prédécesseurs, j'ai dû émettre certaines hypothèses pour dégager cette portion de texte et l'intention qu'elle me paraît représenter. Mathématiquement il s'agit d'une contribution assez cohérente si on la débarrasse de quelques ajouts intempestifs. En revanche il est plus difficile de louer l'articulation produite avec l'analyse de l'arc-en-ciel, puisque nous lui devons sans doute les considérations hors de propos sur la constance

135 V. la restitution que je propose dans l'Annexe III. Il n'y a aucun indice qu'une telle démarche ait été envisagée par l'auteur aristotélicien. 
du rapport HM : MK. Bien entendu, si l'on accepte cette reconstruction, il n'y a plus d'allusion au résultat des Lieux plans dans la portion authentiquement aristotélicienne.

La confrontation de ce chapitre avec la preuve d'Apollonius telle qu'elle est transmise par Eutocius n'est pas toujours très simple dans la mesure où les deux textes semblent altérés à des degrés divers. Paradoxalement c'est le texte du corpus aristotélicien qui permet d'envisager une restauration de la première partie de la démonstration (Annexe III, E), tant l'authenticité apollonienne des détours du texte rapporté par Eutocius paraît improbable. En revanche, pour la seconde partie de ladite preuve (unicité du cercle), nous n'avons pas d'autres ressources que de proposer une solution que rien ne suggère, ni chez Aristote, ni chez Eutocius. Tout au plus, peut-on dire que la solution proposée (construction d'un angle égal à un angle donné, Annexe III, I) n'est qu'une reformulation mathématiquement équivalente au tracé de la tangente au cercle circonscrit au triangle, tracé que mobilise Pappus dans le Lemme qu'il ajoute aux Porismes d'Euclide.

Les caractéristiques communes à ces trois textes mais aussi les irréductibles différences que l'on observe entre le chapitre des Météorologiques et la preuve conservée par Eutocius suggèrent l'hypothèse d'une source commune plutôt qu'une dérivation directe entre ces deux textes. Le Lemme de Pappus incite à penser qu'une telle source pourrait être euclidienne, sans doute un traitement purement géométrique des lieux, comme le recueil des Porismes, plutôt qu'une étude optique que l'on pourrait être tenté de rapporter à la Catoptrique (perdue ou fortement mutilée) de l'Alexandrin. De telles spéculations reviennent à remplacer une hypothèse concernant l'histoire de la géométrie préeuclidienne (Hermotime) que proposaient Tannery et Heath, chacun à leur manière, par une autre, presque aussi incertaine. Mais nous avons vu que le critère des notations complexes n'avait pas le caractère chronologiquement contraignant que lui accordait Tannery. Plus positivement nous avons le témoignage de Pappus qui constitue un lien (ténu) avec les Porismes et l'indication assez sûre qu'Apollonius inscrivait certaines de ses recherches dans la lignée d'Euclide ${ }^{136}$.

136 Sur cette idée d'une filiation intellectuelle entre Euclide et Apollonius, voir [Euclide, 1998], pp. 400-401. 


\section{Bibliographie}

\section{Éditions et traductions de textes anciens}

The first Latin Translation of Euclid's Elements commonly ascribed to Adelard of Bath. H. L. L. Busard (ed.). Toronto, Pontifical Institute of Mediaeval Studies, 1983.

Alexandre d'Aphrodise, Commentaria in Aristotelem Graca, III, 2, M. Hayduck (ed.). Academiæ Litterarum Regiæ Borussicæ. Berlin, G. Reimer 1899. Réimpr. 1960-1961.

Apollonii Pergaei quae Graece exstant cum commentariis antiquis (Eutocius), I-II. J. L. Heiberg (ed.). Leipzig, B. G. Teubner. 1891-1893. Réimpr. Stuttgart, 1974.

Archimedis opera omnia cum commentariis Eutocii, I-III. J. L. Heiberg (ed.). Leipzig, B. G. Teubner, 1910-1915. Réimpr. Stuttgart, 1972.

Aristote, Météorologiques. Traduction française par J. Tricot. Paris, Vrin, 1976.

Aristote, Météorologiques, texte grec établi et traduit en français par P. Louis. Collection des Universités de France, 2 vols. Paris, Belles Lettres, 1982.

Euclid, The Elements. Traduction anglaise et commentaires par T. L. Heath. Cambridge University Press, London, 1908; $2^{\mathrm{e}}$ édition, 1926. Réimpr. New York, Dover Pub., 3 vol., 1956.

Euclide d'Alexandrie, Les Eléments. Traduction française et commentaires par Bernard Vitrac. Paris, PUF, Bibliothèque d'histoire des sciences : Volume 1 : Introduction générale (par Maurice Caveing). Livres I à IV, 1990; Volume 2 : Livres V-IX, 1994; Volume 3 : Livre X, 1998; Volume 4 : Livres XI-XIII, 2001.

Euclidis Elementa, post Heiberg E. S. Stamatis (ed.). Leipzig, B. G. Teubner : I. El. i-iv (1969) ; II. El. v-ix (1970) ; III. El. x (1972) ; IV. El. xi-xiii (1973) ; V,1. El. xiv-xv, Scholia in lib. i-v (1977) ; V, 2. Scholia in lib. vi-xiii (1977).

The Latin translation of the Arabic version of Euclid's Elements commonly ascribed to Gerard of Cremona . H. L. L. Busard (ed). Leiden, E. J. Brill, 1984.

Pappi Alexandrini collectionis quae supersunt. F. Hultsch (ed.). Berlin, Weidmann, 1876-1878 : I. Libr. ii, iii, iv, v, rel. ; II. Libr. vi, vii, rel. ; III. 1. Libr. viii, rel., schol., suppl.; III. 2. indices. Réimpr. Amsterdam, A. M. Hakkert, 1965.

Pappus d'Alexandrie, La collection mathématique. Traduction française P. Ver Eecke. Bruges, 1948. Réimpr. Paris, Blanchard, 1982.

Philon de Byzance, Belopoeica. Edition et traduction anglaise par E. W. Marsden dans : Greek and Roman Artillery. Technical Treatises. Oxford University Press, 1971, pp. 105-155.

Platon, Euvres complètes. Traduction française L. Robin \& J. Moreau. Bibliothèque de la Pléiade. 2 vol. Paris, Gallimard, 1950.

Procli Diadochi in primum Euclidis Elementorum librum Commentarii. G. Friedlein (ed.). Leipzig, B. G. Teubner, 1873. Réimpr. Hildesheim, Georg Olms Verlag, 1967. 
Die Schule des Aristoteles, Texte und Kommentar. F. Wehrli (ed.). Heft VIII : EUDEMOS VON RHODOS. Basel/Stuttgart, Schwabe \& Co 1969.

Die Fragmente der Vorsokratiker. Édition et traduction allemande H. Diels et W. Kranz. 3 vol. 1903. Rév. W. Kranz, 1951. Zürich, Weidmann. Réimpr. Hildesheim, 1985.

\section{Littérature secondaire}

Federspiel, M., "Sur la locution $\epsilon \phi$ ' ổ / '́ $\phi$ ' lettres" dans J. Y. Guillaumin (dir.), Mathématiques dans l'Antiquité. Publications de l'Université de Saint-Étienne, 1992, pp. 9-25.

Granger, G. G, La théorie aristotélicienne de la science. Paris, Aubier Montaigne, 1976.

Heath, T. L., Mathematics in Aristotle. Oxford University Press, 1949. Réimpr. Bristol, Thoemmes Press, 1998.

Knorr, W. R., The Ancient Tradition of Geometric Problems. Boston, Basel, Stuttgart, Birkhäuser, 1986.

Netz, R., The shaping of Deduction in Greek Mathematics. Cambridge University Press, 1999.

Picolet, G., Les sources et le contexte historique de la théorie de l'arc-en-ciel. Thèse, sous la direction de J. Merleau-Ponty, Paris X-Nanterre, 1982. Inédite.

Rommevaux, S., Djebbar, A., Vitrac, B., : "Remarques sur l'histoire du texte des Éléments d'Euclide". Archive for History of Exact Sciences, 55, 2001, pp. 221-295.

Tannery, P. "Le fragment d'Eudème sur la quadrature des lunules". Mémoires de la Société des Sciences physiques et naturelles de Bordeaux, t. V, 1883, pp. 217-237.

Reproduit dans Tannery, P., Mémoires scientifiques. 17 volumes. J. L. Heiberg et H. G. Zeuthen (eds). Paris, Gauthier-Villars, t. I, n²5, 1912, pp. 339-370.

Tannery, P., “Aristote, Météorologie, Livre III, Ch. V”. Revue de philologie, de littérature et d'histoire anciennes, 1886.

Reproduit dans Tannery, P., Mémoires scientifiques. 17 volumes. J. L. Heiberg et H. G. Zeuthen (eds). Paris, Gauthier-Villars, t. IX, n5, 1929, pp. 51-61.

Vitrac, B., "Mythes (et réalités ?) dans l'histoire des mathématiques grecques anciennes" dans L'Europe mathématique. C. Goldstein, J. Gray, J. Ritter (eds). Éditions de la Maison des sciences de l'homme. Paris, 1996, pp. 32-51.

Vitrac, B., "La Définition V. 8 des Eléments d'Euclide". Centaurus, XXXVIII, 2-3, 1996, pp. $97-$ 121.

Vitrac, B., "L'interprétation mathématique du dilemme du cône (DK 68 B 155). Démocrite était-il mathématicien ?" dans Les anciens savants. Études sur les philosophies préplatoniciennes réunies par Pierre-Marie Morel et Jean-François Pradeau. Presses Universitaires de Strasbourg, à paraître 2002, pp. 85-125. 


\section{Annexes}

I

Pappus, Collection mathématique, Livre VII, § XXIX, Proposition $155=$

$28^{\mathrm{e}}$ Lemme pour le second Porisme du Premier Livre des Porismes d'Euclide.

(Ed. Hultsch, p. 904, 1. 17-p. 906, 1. 12).

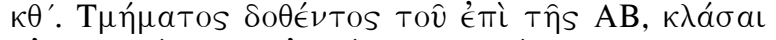

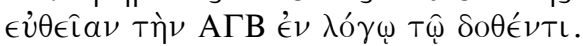

Un segment de cercle sur la droite $\mathrm{AB}$ étant donné, fléchir la ligne ACB dans un rapport donné.
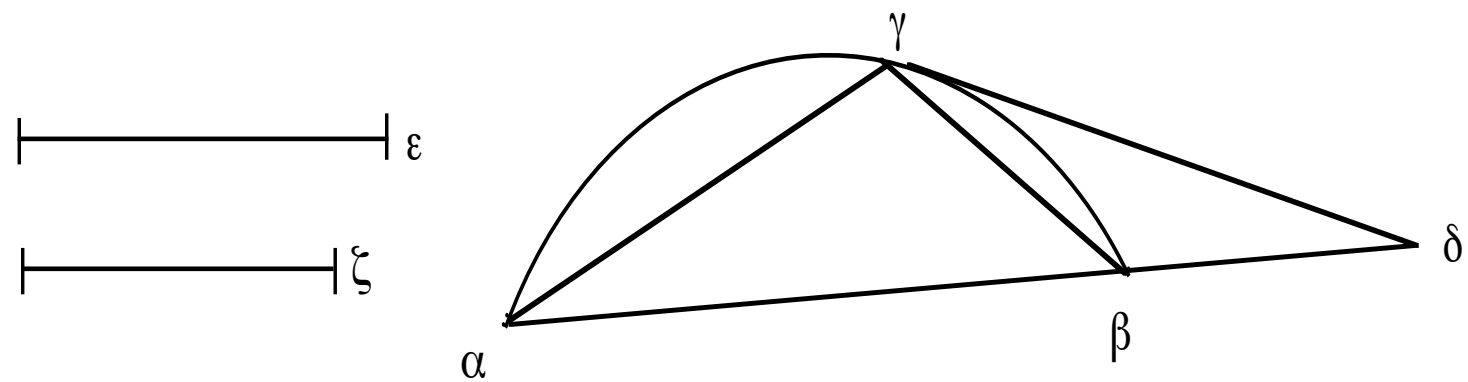

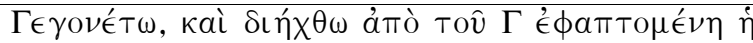

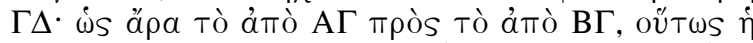

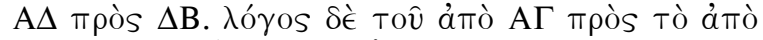

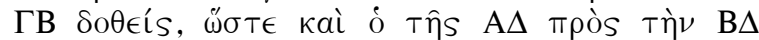

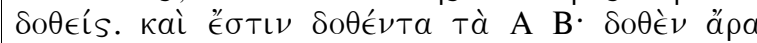

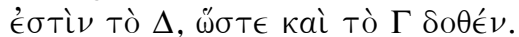

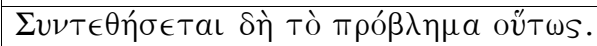

Qu'il en soit ainsi et qu'à partir de $\Gamma$ soit menée la tangente $\Gamma \Delta$; donc comme le carré sur $\mathrm{A} \Gamma$ est relativement à celui sur $\mathrm{B} \Gamma$, ainsi est $\mathrm{A} \Delta$ relativement à $\Delta \mathrm{B}^{137}$. Or le rapport du carré sur $\mathrm{A} \Gamma$ relativement à celui sur $\Gamma \mathrm{B}$ est donné, de sorte que le rapport de $\mathrm{A} \Delta$ relativement à $\mathrm{B} \Delta$ est aussi donné. Et les points $\mathrm{A} \mathrm{B}$ sont donnés. Donc $\Delta$ est donné, de sorte que $\Gamma$ est aussi donné.

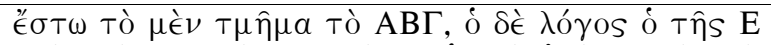

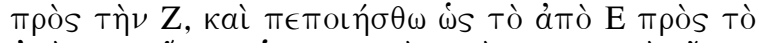

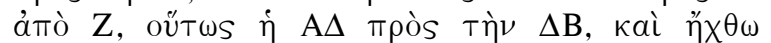

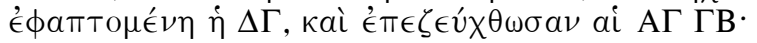

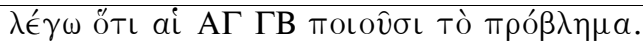
Le problème sera alors synthétisé ainsi :

Soit d'une part le segment $\mathrm{AB} \Gamma$, d'autre part le rapport, celui de E relativement à $Z$, et qu'il soit fait que comme le carré sur E est relativement à celui sur $\mathrm{Z}$, ainsi soit $\mathrm{A} \Delta$ relativement à $\Delta \mathrm{B}$, et que soit menée la tangente $\Delta \Gamma$, et que les droites $\mathrm{A} \Gamma \Gamma \mathrm{B}$ soient jointes.

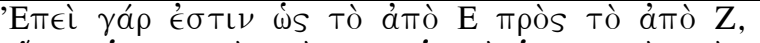
Je dis que les droites $\mathrm{A} \Gamma \Gamma \mathrm{B}$ réalisent le problème.

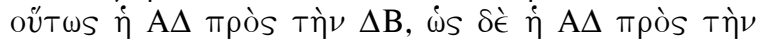

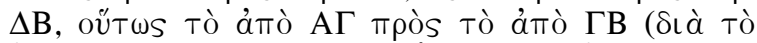

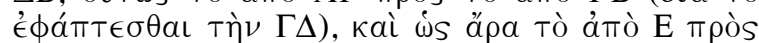
Tò åmò $\mathrm{Z}$, oútws Tò ámò $\mathrm{A} \Gamma \pi \rho$ òs Tò ámò $\Gamma \mathrm{B}$.

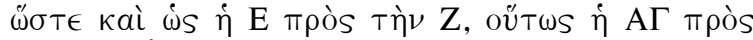

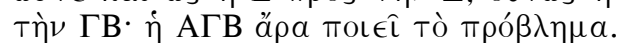

En effet, puisque comme le carré sur $\mathrm{E}$ est relativement à celui sur $\mathrm{Z}$, ainsi est $\mathrm{A} \Delta$ relativement à $\Delta \mathrm{B}$, que comme $\mathrm{A} \Delta$ est relativement à $\Delta \mathrm{B}$, ainsi est le carré sur $\mathrm{A} \Gamma$ relativement à celui sur $\Gamma \mathrm{B}$ (parce que $\Gamma \Delta$ est tangente), donc, aussi, comme le carré sur $\mathrm{E}$ est relativement à celui sur $\mathrm{Z}$, ainsi est le carré sur $\mathrm{A} \Gamma$ relativement à celui sur $\Gamma \mathrm{B}$; de sorte qu'aussi, comme $\mathrm{E}$ est relativement à $\mathrm{Z}$, ainsi est $\mathrm{A} \Gamma$ relativement à $\Gamma \mathrm{B}$; donc la ligne $\mathrm{A} \Gamma \mathrm{B}$ réalise le problème.

137 Puisque $\Gamma \Delta$ est tangente, l'angle sous $\mathrm{B} \Gamma \Delta$ est égal à celui sous $\Gamma A \Delta$ (Eucl. III. 32). Et puisque les triangles $\mathrm{B} \Gamma \Delta$ et $\Gamma A \Delta$ ont aussi l'angle $\Delta$ en commun, ils sont équiangles (Eucl. I. 32), donc semblables. D'où : $\mathrm{A} \Delta: \Gamma \Delta:: \Gamma \Delta: \mathrm{B} \Delta:: \mathrm{A} \Gamma: \mathrm{B} \Gamma$ et donc $: \mathrm{A} \Delta: \mathrm{B} \Delta:: \mathrm{A} \Gamma^{2}: \mathrm{B} \Gamma^{2}$. 
Apollonius, Extrait des Lieux plans (?) d'après Eutocius, Commentaires aux Coniques

Ed. Heiberg, II, p. 180, 1. 11-p. 184, 1. 20

\begin{tabular}{|c|c|c|}
\hline & 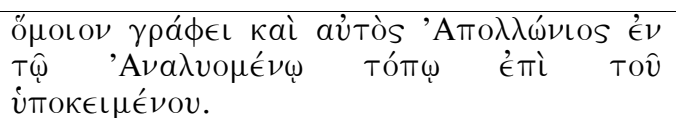 & $\begin{array}{l}\text { Et Apollonius lui-même écrit de la même manière sur } \\
\text { ce sujet dans le Lieu analysé }\end{array}$ \\
\hline $\begin{array}{c}\text { Énoncé } \\
138\end{array}$ & 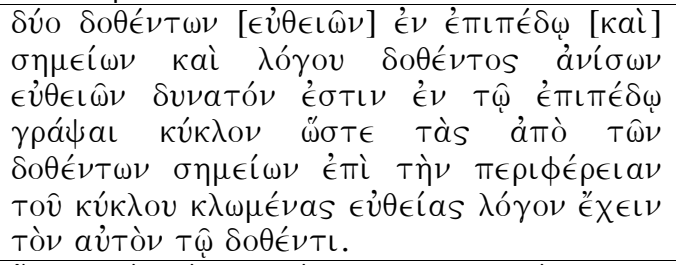 & $\begin{array}{l}\text { Deux points étant donnés et un rapport donné entre } \\
\text { deux droites inégales, il est possible de décrire un } \\
\text { cercle dans le plan de sorte que les droites fléchies à } \\
\text { partir des points donnés jusqu'à la circonférence du } \\
\text { cercle aient le même rapport que le rapport donné. }\end{array}$ \\
\hline $\begin{array}{c}\text { (B) } \\
\text { Ecthèse }\end{array}$ & 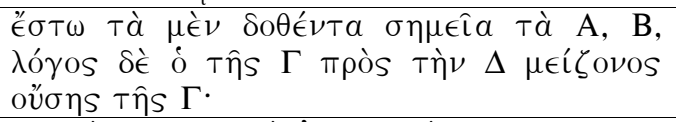 & $\begin{array}{l}\text { Soit d'une part } \mathrm{A}, \mathrm{B}, \text { les points donnés, d'autre part le } \\
\text { rapport, celui de } \Gamma \text { à } \Delta, \Gamma \text { étant la plus grande. }\end{array}$ \\
\hline (C) Diorisme & 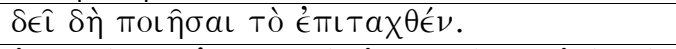 & Il faut alors faire ce qui est prescrit. \\
\hline $\begin{array}{c}\text { (D) } \\
\text { Construction } \\
\text { VI. } 11\end{array}$ & 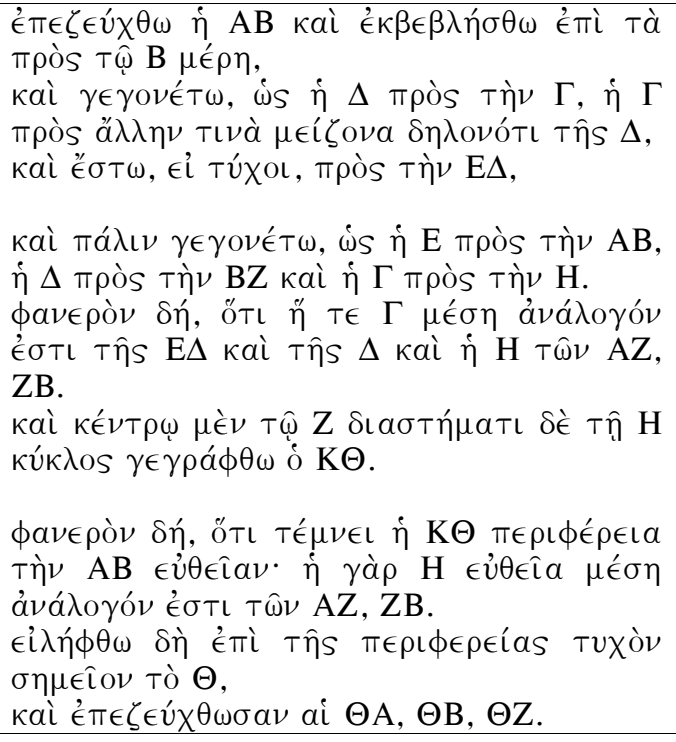 & $\begin{array}{l}\text { Que } \mathrm{AB} \text { soit jointe et prolongée du côté de } \mathrm{B} \text {. } \\
\text { Et qu'il soit fait que comme } \Delta \text { est à } \Gamma \text {, ainsi soit } \Gamma \\
\text { relativement à une autre droite, manifestement plus } \\
\text { grande que } \Delta \text {, et que ce soit, par exemple, relativement } \\
\text { à } \mathrm{E}+\Delta \\
\text { Et, de nouveau, qu'il soit fait que comme } \mathrm{E} \text { est à } \mathrm{AB} \text {, } \\
\text { ainsi soit } \Delta \text { relativement à } \mathrm{BZ} \text { et } \Gamma \text { relativement à } \mathrm{H} \\
\text { Il est alors évident que } \Gamma \text { est moyenne proportionnelle } \\
\text { de } \mathrm{E}+\Delta \text { et } \Delta \text {, et } \mathrm{H} \text { celle des droites } \mathrm{AZ}, \mathrm{ZB} \text {. } \\
\text { Et d'une part avec } \mathrm{Z} \text { comme centre, d'autre part avec } \mathrm{H} \\
\text { comme intervalle, que soit décrit le cercle } \mathrm{K} \Theta \text {. } \\
\text { Il est alors évident que la circonférence } \mathrm{K} \Theta \text { coupe la } \\
\text { droite AB parce que la droite } \mathrm{H} \text { est moyenne } \\
\text { proportionnelle des droites } \mathrm{AZ}, \mathrm{ZB} \mathrm{B}^{139} \text {. } \\
\text { Que soit alors pris au hasard un point } \Theta \text { sur la } \\
\text { circonférence. } \\
\text { Et que soient jointes } \Theta \mathrm{A}, \Theta \mathrm{B}, \Theta Z \text {. }\end{array}$ \\
\hline & $\Gamma$ & 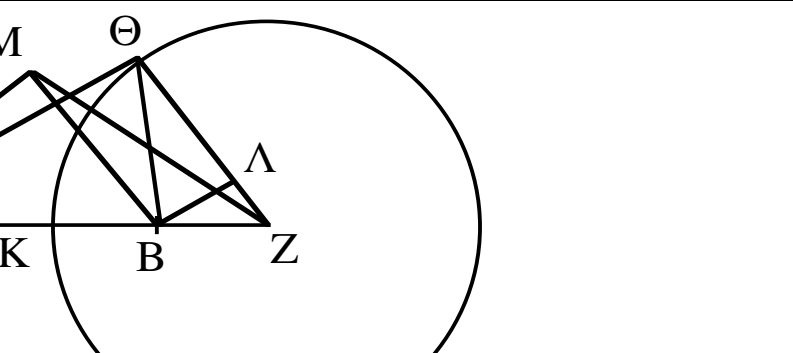 \\
\hline $\begin{array}{c}\mathbf{E}) \\
\text { Démonstrat. }\end{array}$ & 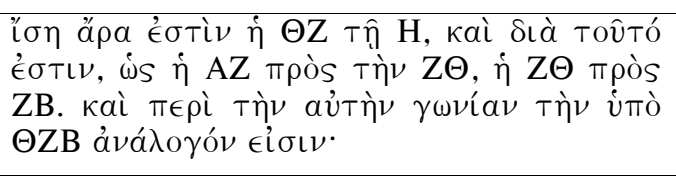 & $\begin{array}{l}\text { Donc } \Theta Z \text { est égale à } H \text {. Et, à cause de cela, comme } A Z \\
\text { est relativement à } \Theta Z \text {, ainsi est } Z \Theta \text { relativement à } Z B \text {. } \\
\text { Et il y a proportion autour du même angle, celui sous } \\
\text { OZB. }\end{array}$ \\
\hline
\end{tabular}

${ }^{138}$ L'énoncé était peut-être double, à cause du diorisme intermédiaire introduisant la démonstration d'unicité; V. infra, Annexe III, A.

${ }^{139}$ Cette phrase pour faire appel à l'évidence à l'aide d'une explication postposée triviale est certainement inauthentique. 


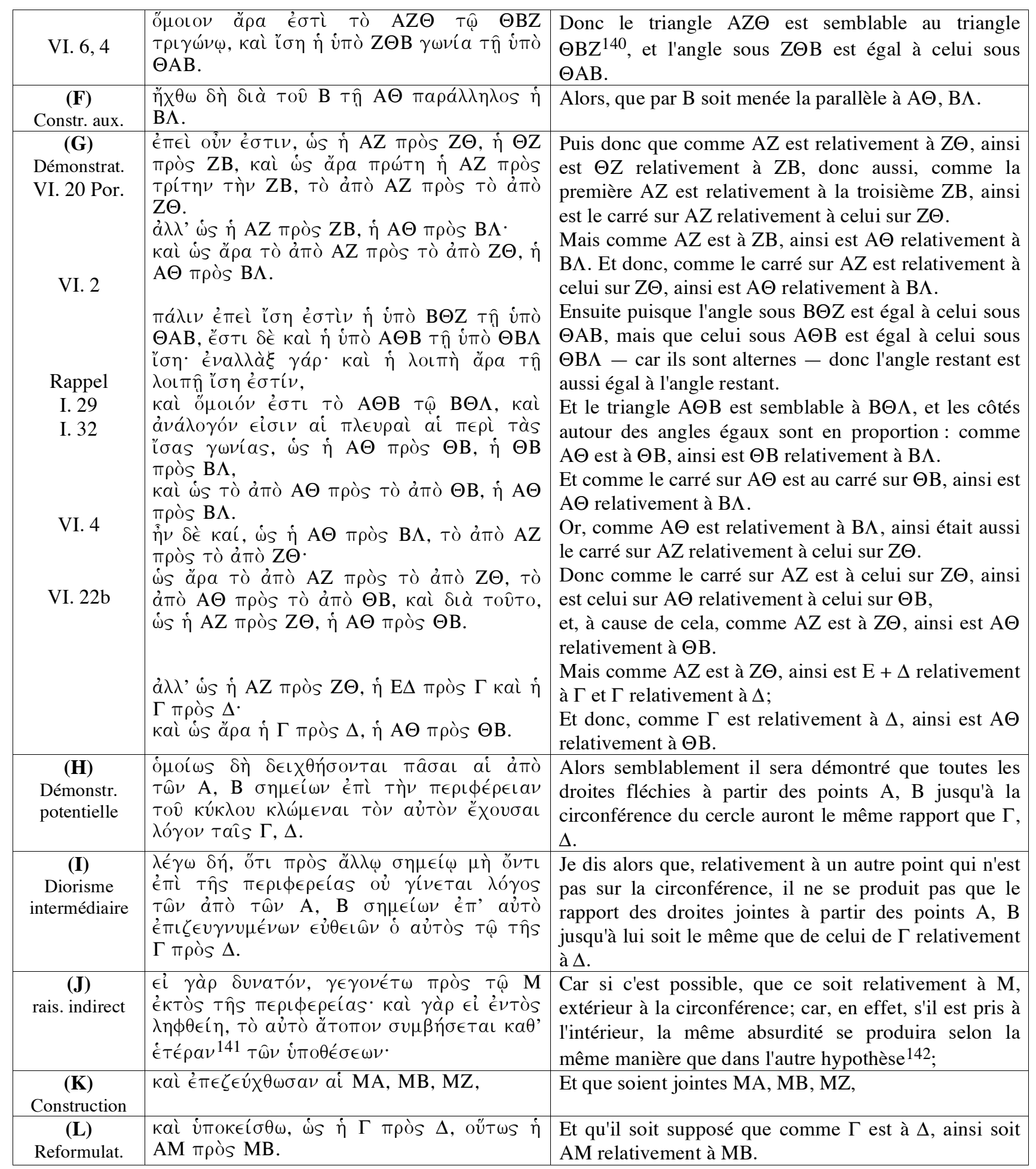

140 A partir de cette similitude des triangles AZ $\Theta$ et $\Theta B Z$ il était facile de conclure. V. infra, Annexe III, E (la portion restituée est mise en italiques). Le texte transmis par Eutocius est inutilement compliqué. Il comporte plusieurs rappels très élémentaires pour des assertions obtenues juste auparavant; sans doute s'agit-il d'un remaniement.

${ }^{141}$ Dans les mss "Éкaté $\rho a \nu "$ : chacune (des hypothèses).

142 Cette justification postposée pour écarter d'emblée un second cas de figure n'est pas canonique, non plus que sa formulation plutôt métamathématique sur «l'identité des absurdités ». Généralement on trouve, à la fin du raisonnement indirect portant sur l'une des branches de l'alternative une démonstration potentielle expéditive pour indiquer que l'autre serait « semblablement démontrée ». V. infra, Annexe III, L. 


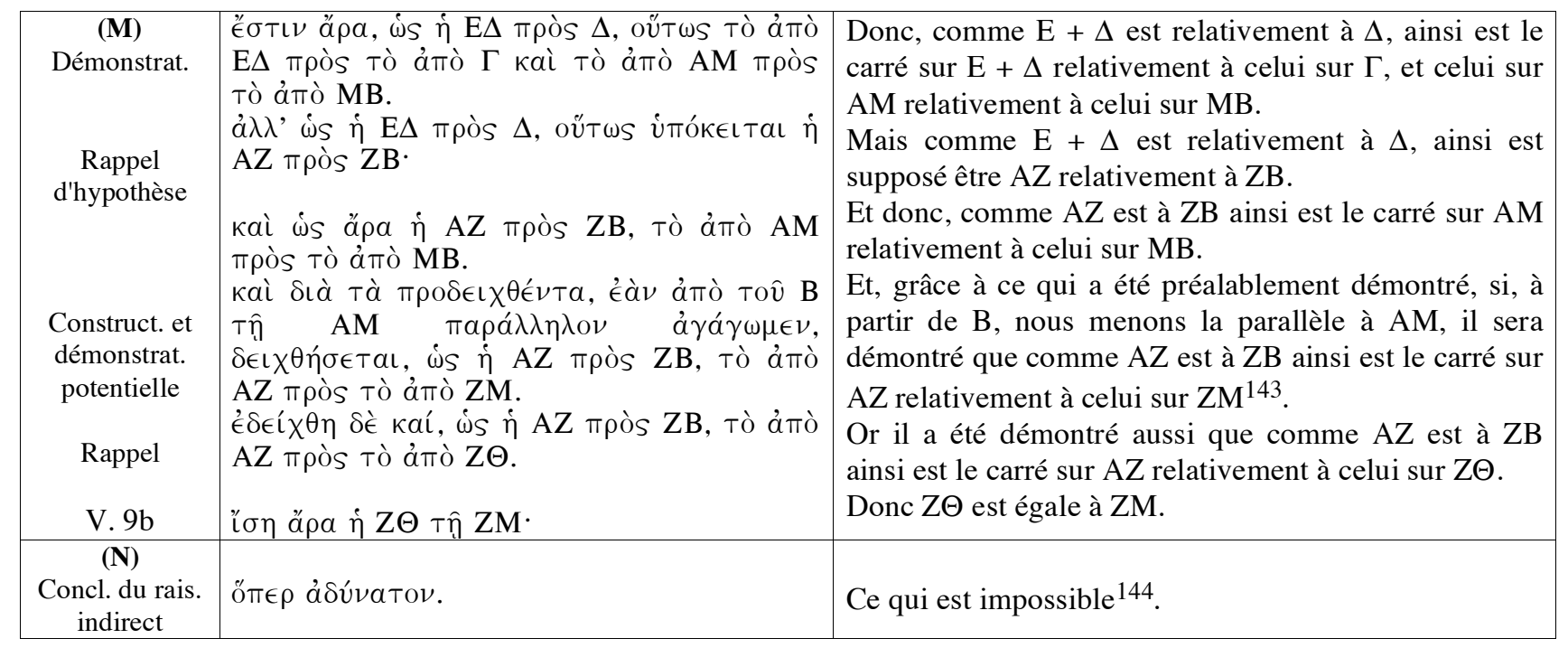

\section{III}

Tentative de restitution de la synthèse d'Apollonius

\begin{tabular}{|c|c|c|}
\hline $\begin{array}{l}\text { Énoncé } \\
\text { double }\end{array}$ & 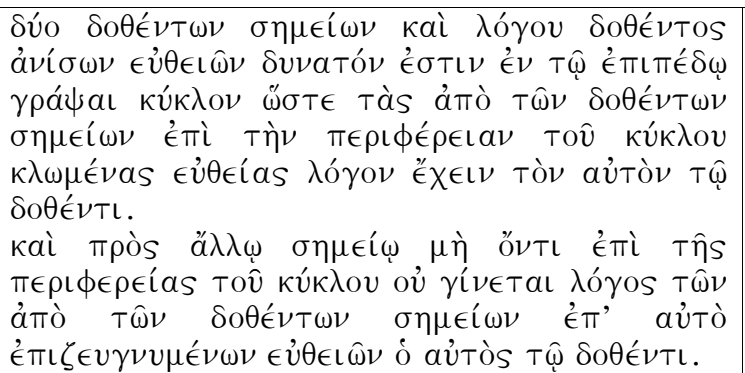 & $\begin{array}{l}\text { Deux points étant donnés et un rapport donné entre } \\
\text { deux droites inégales, il est possible de décrire un } \\
\text { cercle dans le plan de sorte que les droites fléchies à } \\
\text { partir des points donnés jusqu'à la circonférence du } \\
\text { cercle aient le même rapport que le rapport donné. } \\
\text { Et relativement à un autre point qui n'est pas sur la } \\
\text { circonférence du cercle, il ne se produit pas que le } \\
\text { rapport des droites jointes à partir des points donnés } \\
\text { jusqu'à celui-ci soit le même que le rapport donné. }\end{array}$ \\
\hline (B) & 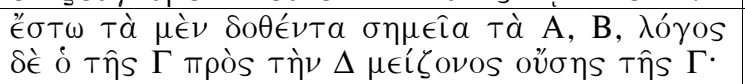 & $\begin{array}{l}\text { Soit d'une part } \mathrm{A}, \mathrm{B} \text {, les points donnés, d'autre part } \\
\text { le rapport, celui de } \Gamma \text { à } \Delta, \Gamma \text { étant la plus grande. }\end{array}$ \\
\hline (C) & 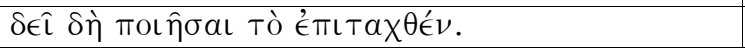 & Il faut alors faire ce qui est prescrit. \\
\hline VI. 12 & 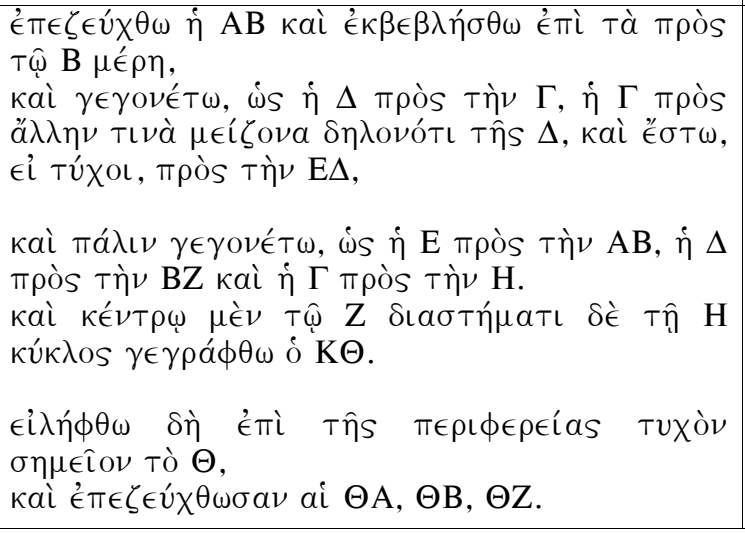 & $\begin{array}{l}\text { Que } \mathrm{AB} \text { soit jointe et prolongée du côté de } \mathrm{B} \text {. } \\
\text { Et qu'il soit fait que comme } \Delta \text { est à } \Gamma \text {, ainsi soit } \Gamma \\
\text { relativement à une autre droite, manifestement plus } \\
\text { grande que } \Delta \text {, et que ce soit, par exemple, } \\
\text { relativement à } \mathrm{E}+\Delta \\
\text { Et, de nouveau, qu'il soit fait que comme E est à } \\
\mathrm{AB} \text {, ainsi soit } \Delta \text { relativement à } \mathrm{BZ} \text { et } \Gamma \text { relativement } \\
\text { à } \mathrm{H} \text {. } \\
\text { Et d'une part avec } \mathrm{Z} \text { comme centre, d'autre part avec } \\
\mathrm{H} \text { comme intervalle, que soit décrit le cercle } \mathrm{K} \Theta \text {. } \\
\text { Que soit alors pris au hasard un point } \Theta \text { sur la } \\
\text { circonférence. } \\
\text { Et que soient jointes } \Theta \mathrm{A}, \Theta \mathrm{B}, \Theta Z \text {. }\end{array}$ \\
\hline
\end{tabular}

143 La proportion « comme $\mathrm{AZ}$ est à $\mathrm{ZB}$ ainsi est le carré sur $\mathrm{AZ}$ relativement à celui sur $\mathrm{Z} \Theta$ » est introduite dans $(\mathbf{G})$ donc après le tracé de la parallèle $\mathrm{B} \Lambda$ ! Mais c'est une conséquence (via VI. 20 Por) de la proportion « comme $\mathrm{AZ}$ est à $\mathrm{Z} \Theta$ ainsi est $\Theta Z$ relativement à $Z B$ », c'est-à-dire de l'hypothèse que $Z \Theta$ est prise égale à $H$, moyenne proportionnelle

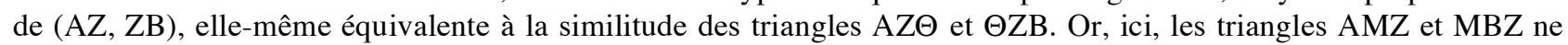
sont pas semblables et il n'est pas supposé que MZ soit moyenne proportionnelle de (AZ, ZB). Je ne vois donc pas comment conclure de cette manière. La démonstration consiste sans doute à introduire le point $\mathrm{Z}$ de telle manière que l'angle sous BMZ soit égal à l'angle MAB afin que, comme précédemment, les triangles AMZ et MBZ soient encore équiangles, donc semblables. MZ sera alors moyenne proportionnelle de (AZ, ZB). On sera ainsi ramené à la situation précédente et le point $\mathrm{Z}$, ainsi construit, sera le centre du cercle décrit dans la première partie. V. infra, Annexe III, K. 144 On peut considérer qu'il manque une conclusion et vouloir l'ajouter (V. infra, Annexe III, M). Il s'agit d'une simple intervention cosmétique. 


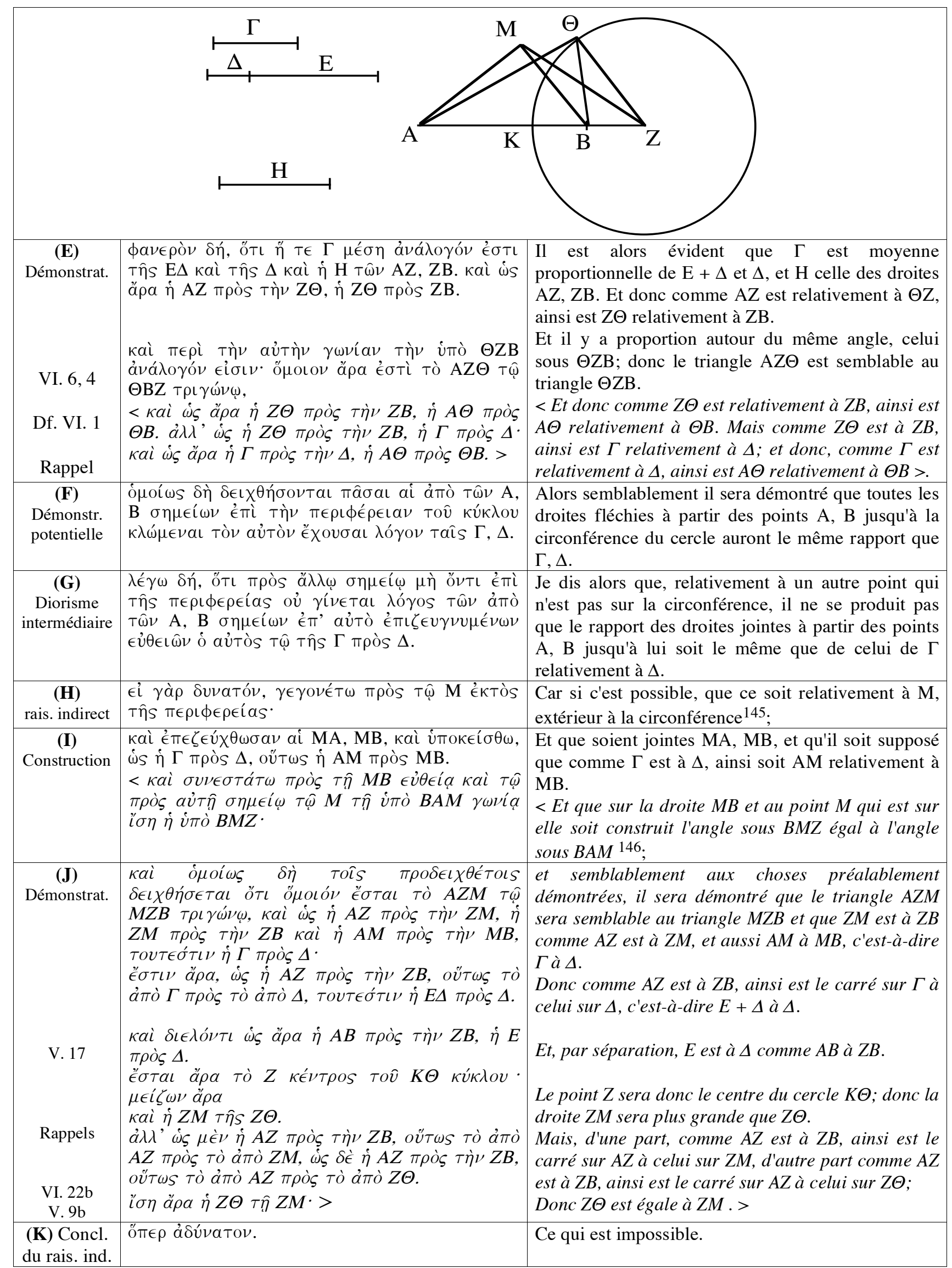

$145 \mathrm{~J}$ 'ai maintenu la forme indirecte du raisonnement pour aboutir à une contradiction $(\mathrm{ZM}>\mathrm{Z} \Theta ; \mathrm{ZM}=\mathrm{Z \Theta})$.

${ }^{146} \mathrm{Si}$ l'on veut rester plus proche de Pappus on peut aussi décrire le cercle circonscrit au triangle AMB, tracer la tangente en $\mathrm{M}$ qui coupe le prolongement de $\mathrm{AB}$ en $\mathrm{Z}$... Cela revient au même mais a l'inconvénient d'introduire une opération de plus. 


\begin{tabular}{|c|c|c|}
\hline $\begin{array}{l}\text { (L) Dém. } \\
\text { potentielle }\end{array}$ & 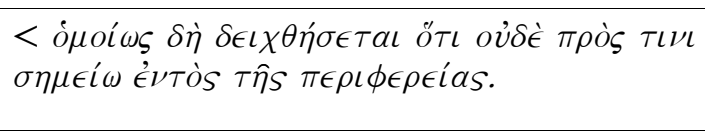 & $\begin{array}{l}\text { < Alors semblablement il sera démontré que ce n'est } \\
\text { pas le cas non plus relativement à quelque point } \\
\text { intérieur à la circonférence; }\end{array}$ \\
\hline Conclusion & 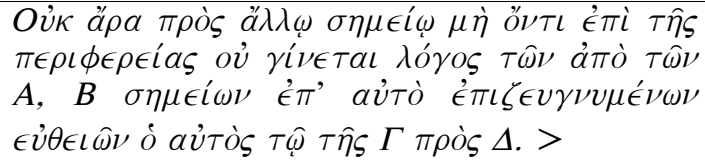 & $\begin{array}{l}\text { Donc, relativement à un autre point qui n'est pas sur } \\
\text { la circonférence, il ne se produit pas que le rapport } \\
\text { des droites jointes à partir des points A, B jusqu'à lui } \\
\text { soit le même que de celui de } \Gamma \text { relativement à } \Delta .>\end{array}$ \\
\hline
\end{tabular}

